\title{
Treating depression and depression-like behavior with physical activity: an immune perspective
}

\author{
Harris A. Eyre ${ }^{1,2}$, Evan Papps ${ }^{1}$ and Bernhard T. Baune ${ }^{1 *}$ \\ ${ }^{1}$ Discipline of Psychiatry, School of Medicine, University of Adelaide, Adelaide, SA, Australia \\ ${ }^{2}$ School of Medicine and Dentistry, James Cook University, Townsville, QLD, Australia
}

\author{
Edited by: \\ Felipe Schuch, Hospital de Clínicas de \\ Porto Alegre, Brazil \\ Reviewed by: \\ Oliver Grimm, Central Institute of \\ Mental Health, Germany \\ Mark Horowitz, King's College \\ London, UK \\ Bianca W. De Aguiar, Universidade \\ Federal do Rio Grande do Sul, Brazil \\ *Correspondence: \\ Bernhard T. Baune, Discipline of \\ Psychiatry, School of Medicine, \\ University of Adelaide, Adelaide, SA \\ 5005, Australia. \\ e-mail: bernhard.baune@ \\ adelaide.edu.au
}

The increasing burden of major depressive disorder makes the search for an extended understanding of etiology, and for the development of additional treatments highly significant. Biological factors may be useful biomarkers for treatment with physical activity (PA), and neurobiological effects of PA may herald new therapeutic development in the future. This paper provides a thorough and up-to-date review of studies examining the neuroimmunomodulatory effects of PA on the brain in depression and depression-like behaviors. From a neuroimmune perspective, evidence suggests PA does enhance the beneficial and reduce the detrimental effects of the neuroimmune system. PA appears to increase the following factors: interleukin (IL)-10, IL-6 (acutely), macrophage migration inhibitory factor, central nervous system-specific autoreactive CD4+ T cells, M2 microglia, quiescent astrocytes, CX3CL1, and insulin-like growth factor-1. On the other hand, PA appears to reduce detrimental neuroimmune factors such as: Th1/Th2 balance, pro-inflammatory cytokines, C-reactive protein, M1 microglia, and reactive astrocytes. The effect of other mechanisms is unknown, such as: CD4+CD25+ T regulatory cells (T regs), CD200, chemokines, miRNA, M2-type blood-derived macrophages, and tumor necrosis factor (TNF)- $\alpha$ [via receptor 2 (R2)]. The beneficial effects of PA are likely to occur centrally and peripherally (e.g., in visceral fat reduction). The investigation of the neuroimmune effects of PA on depression and depression-like behavior is a rapidly developing and important field.

Keywords: physical activity, exercise, depression, psychiatry, immune, neurobiology
The increasing burden of major depressive disorder (MDD; WHO, 2008) makes the search for an extended understanding of etiology, and for the development of additional treatments highly significant. The global "pandemic" of physical inactivity (Lee et al., 2012) - a significant etiological factor for many noncommunicable diseases, including depression (Garber et al., 2011; Kohl et al., 2012; Lee et al., 2012) - as well as the growing evidence supporting the clinical utility of physical activity (PA) in many psychiatric disorders, make the biological effects of PA highly relevant (Knochel et al., 2012; Lautenschlager et al., 2012; Rimer et al., 2012). Biological factors may be useful biomarkers for treatment with PA, and neurobiological effects of PA may herald new therapeutic developments in the future.

The neuroimmune system is important in the pathogenesis and pathophysiology of depression-like behaviors (Eyre and Baune, 2012c). Elevations in pro-inflammatory cytokines (PICs), causing neuroinflammation, are well known to be involved in the development of depression-like behaviors - e.g., sickness-like behavior, cognitive dysfunction, and anhedonia - in pre-clinical and clinical populations (Dantzer et al., 2008; McAfoose and Baune, 2009; Miller et al., 2009). The involvement of PICs in the development of depression-like behavior is often referred to as the cytokine model of depression (Dantzer et al., 2008; McAfoose and Baune, 2009; Miller et al., 2009).The neuroinflammatory state is associated with neurotransmitter dysfunction [e.g., reductions in serotonin
(5-HT), as well as neurotoxic levels of glutamate (GLU) and tryptophan catabolites], reduced hippocampal (HC) neuroplasticity [e.g., neurogenesis, synaptic plasticity, and long-term potentiation (LTP)], oxidative stress, and glucocorticoid insensitivity (Dantzer et al., 2008; Miller et al., 2009; Eyre and Baune, 2012c; Leonard and Maes, 2012; Moylan et al., 2012).

A variety of novel neuroimmune mechanisms may also be involved in the development of depression-like behaviors (Eyre and Baune, 2012c; Littrell, 2012). Cellular immune factors include various $\mathrm{T}$ cells [e.g., CD4+CD25+ T regulatory cells ( $\mathrm{T}$ regs), CNS-specific autoreactive CD4+ T cells] and macrophages (e.g., M2-type blood-derived macrophages) involved in the model of protective immunosurveillance (Schwartz and Shechter, 2010a,b; Martino et al., 2011; Ron-Harel et al., 2011). These neuroprotective immune cells - found to release neurotrophic factors and antiinflammatory cytokines (AICs; Schwartz and Shechter, 2010a,b; Martino et al., 2011; Ron-Harel et al., 2011) - may be dysfunctional in the disease state (Schwartz and Shechter, 2010b). Moreover, the function of immunomodulatory proteins such as CX3CL1 (aka fractalkine; Rogers et al., 2011; Corona et al., 2012; Giunti et al., 2012), insulin-like growth factor-1 (IGF-1; Park et al., 2011a), and CD 200 (Lyons et al., 2007; Ojo et al., 2012) may be reduced.

In clinical studies, PA has shown efficacy in the treatment of MDD (Rimer et al., 2012), schizophrenia (SCZ; Knochel et al., 2012), anxiety-based disorders (Asmundson et al., 2013), and in 
enhancing cognitive function in disorders of cognitive function (i.e., Alzheimer's disease, $\mathrm{AD}$ and mild cognitive impairment, MCI; Foster et al., 2011; Knochel et al., 2012; Lautenschlager et al., 2012). There are many reasons why PA is an attractive therapeutic option in psychiatry. It has a low side-effect profile and can be adapted according to a patient's medical co-morbidities and functional status (Garber et al., 2011; Knochel et al., 2012; Rimer et al., 2012). PA also enhances self-esteem (Salmon, 2001), has less stigmatization than psychotherapy, may reduce the use of pharmacotherapies in MDD (Deslandes et al., 2010) and has a positive effect on cardiometabolic risk factors relevant to many psychiatric diseases (e.g., chronic inflammation, visceral fat mass, glucocorticoid sensitivity, glucose control, and insulin sensitivity; Gleeson et al., 2011; Baune et al., 2012c; Hamer et al., 2012; Knochel et al., 2012; Stuart and Baune, 2012).

Physical activity has beneficial effects on depressive symptomatology in a variety of clinical contexts. It is found to have robust effects on the depressive phenotype found in MDD (Rimer et al., 2012), as well as beneficial effects on the depressive symptomatology involved in the negative symptoms of SCZ (Knochel et al., 2012). PA has also been shown to be effective in treating cognitive dysfunction-related depression (Knochel et al., 2012; i.e., in MCI and $\mathrm{AD}$ where a significant proportion of patients with $\mathrm{AD}$ suffer from co-morbid depression; Lee and Lyketsos, 2003). The clinical utility of PA in MDD is promising given most patients on antidepressants will not achieve remission following initial treatment (Trivedi et al., 2006), and nearly one-third will not achieve remission even following several treatment steps (Rush et al., 2006a,b). Encouragingly, a recent Cochrane meta-analysis of 28 trials (1101 participants) by Rimer et al. (2012) - comparing exercise with no treatment or control intervention - found a moderate clinical effect in MDD. Studies have found that whilst PA has an initial treatment effect equal to that of antidepressants (Rimer et al., 2012), its effects are slower (Blumenthal et al., 1999) with greater relapse prevention (Babyak et al., 2000). PA interventions have been shown to be efficacious as a stand-alone (Rethorst et al., 2009) and as an augmentation treatment for MDD (Trivedi et al., 2011). Adequate levels of PA are also found to have a role in the prevention of MDD (Pasco et al., 2011b).

Physical activity interventions are found to have a multitude of effects on neuroimmune processes (Eyre and Baune, 2012a). Most notably PA interventions are found to reduce PIC levels in the brain of rodents (Eyre and Baune, 2012a) and in the periphery in clinical studies (Beavers et al., 2010a; Rethorst et al., 2012). The anti-inflammatory effects of PA may be related to acute elevations in neuroprotective interleukin-6 (IL-6; Funk et al., 2011), and resultant downstream changes, e.g., increased IL-1ra and reduced neuronal death in the HC (Funk et al., 2011). Reductions in pro-inflammatory visceral fat mass may also play a role in the anti-inflammatory effect of PA (Gleeson et al., 2011).

The neuroimmune effects of PA were recently outlined in our review (Eyre and Baune, 2012a), however, there have been a large number of studies published in 2012 investigating other neuroimmune-related factors (Moon et al., 2012; Rethorst et al., 2012). Novel factors investigated include macrophage migration inhibitor factor (MIF; Moon et al., 2012), CX3CL1 (Vukovic et al., 2012), and IGF-1 (Duman et al., 2009). Taken together, there is a need for a review outlining and summarizing these recent studies in light of pre-existing literature with the intention of better understanding the neuroimmunological effects of PA. From this literature important questions arise: Are there PA types which are more effective than others? Are there subpopulations of patients with MDD who would benefit more from PA than antidepressants or psychotherapy? Can the neuroimmune effects of PA inform therapeutic development in the future? Are immune biomarkers potentially useful in measuring a treatment effect for PA in depression?

This paper provides a thorough and up-to-date review of studies examining the neuroimmunomodulatory effects of PA on the brain in depression and depression-like behaviors.

\section{METHODS}

This review utilized an electronic search of databases such as PubMed, PsychInfo, OvidSP, and Science Direct. An initial search was conducted using the following keywords: (PA OR exercise) AND (immune OR inflammation OR cytokine OR antiinflammatory OR immune cell OR glia OR neuroplasticity) AND/OR depression. Abstracts were selected based on the year of publication (between 1995 and December 2012), publication in the English language and of peer-reviewed type. They were excluded if they included anecdotal evidence. A total of 16,000 studies were found using these search terms. A total of 1000 articles remained after assessment of abstracts for relevance to the aims of this review. Of these, 770 studies were excluded after review of the full text if they did not examine the effect of the PA or depression on the immune system. A proportion of papers were found via the reference lists of the 1000 full text articles. Finally, 230 articles were utilized in this review.

\section{CLINICAL EFFICACY OF PHYSICAL ACTIVITY IN DEPRESSION}

Evidence supporting the clinical efficacy of PA interventions with depression - and depression co-morbid with other diseases [MCI, coronary heart disease (CHD)] - is growing (Blumenthal et al., 2012a,b; Rimer et al., 2012). In the clinical setting, exercise interventions are defined as "planned, structured, and repetitive bodily movements done to improve or maintain one or more components of physical fitness" (Garber et al., 2011). Exercise types can include aerobic, resistance, neuromotor (involving balance, agility, and co-ordination), and flexibility types (Garber et al., 2011). The following section will outline clinical evidence supporting the use of exercise in depression.

A 2012 re-analysis of available clinical trials by the Cochrane Group (Rimer et al., 2012; 2009 version; Mead et al., 2008) revealed 28 trials (1101 participants) comparing exercise with no treatment or control intervention finding a moderate clinical effect in MDD (standardized mean difference, SMD; -0.67 95\% CI -0.90 to -0.43$)$. However, when the meta-analysis was conducted with more strict criteria - i.e., studies with adequate allocation concealment, intention-to-treat analysis, and blinded outcome assessment - there were only four trials (326 participants), the SMD indicated a small clinical effect (SMD $-0.3195 \%$ CI -0.63 to 0.01 ). Moreover, data from the seven trials (373 participants) that provided long-term follow-up also found a small effect for exercise interventions (SMD $-0.39,95 \% \mathrm{CI}-0.69$ to -0.09 ). 
In comparison to cognitive behavioral therapy, six trials (152 participants) found no significant difference with exercise.

Further investigating the individual clinical trials analyzed in this field yields interesting information on the clinical effect of exercise regimens. A 16-week randomized controlled trial (RCT) study by Blumenthal et al. (1999) found aerobic exercise and antidepressant (sertraline) treatment were equally effective in reducing depressive symptom severity [as per both Hamilton Depression Rating Scale (HAM-D) and Beck Depression Inventory (BDI)], however, sertaline had a faster initial response (in the first 3 weeks). Shortly after, a paper by Babyak et al. (2000) was published on the same study participants showing - at 6 months follow-up patients assigned to the exercise program were less likely to relapse (no longer diagnostic for MDD or HAM-D < 8) than patients assigned to antidepressant treatment. Self-initiated exercise after the study intervention was associated with a reduced probability of depression at the end of the follow-up period ( $\mathrm{OR}=0.49)$.

Treatment of depression in older people is often hampered by poor recognition and increased prevalence of medication sideeffects, polypharmacy, and poor adherence to treatment; therefore, exercise is increasingly being evaluated as a possible treatment. A recent meta-analysis (Bridle et al., 2012) of seven trials of subjects $\geq 60$ years found exercise was associated with significantly lower depression severity (SMD $-0.34 ; 95 \% \mathrm{CI}-0.52$ to -0.17 ). These findings were irrespective of whether participant eligibility was determined by clinical diagnosis or symptom checklist. An RCT in elderly patients ( $>60$ years) with MDD - non-responders to escitalopram - found a 10-week Tai Chi Chih (TCC) exercise intervention augmented antidepressant treatment (Lavretsky et al., 2011). TCC exercise was chosen given it can be readily implemented among older adults with physical limitations (due to chronic medical illnesses or poor balance) and its added stress reduction and mindful cognitive properties. Multiple studies have shown regular, moderate PA can have a positive influence on depressive symptomatology in subjects with AD (Knochel et al., 2012), however Mahendra and Arkin (2003) found this beneficial effect was only significant after $>1$ year of PA. Deslandes et al. (2010) reported patients with co-morbid MCI and MDD could significantly reduce their antidepressant dose when they underwent a PA program.

Exercise is shown to have some modest beneficial effects on certain aspects of neurocognitive disturbance in depression. An RCT study with patients who met MDD criteria found exercise (both supervised and home-based) performed better with exercise than sertraline on tests of executive functioning, but not on tests of verbal and working memory (Hoffman et al., 2008). A recent meta-analysis (Smith et al., 2010) examining the effects of aerobic exercise on neurocognitive performance found 29 studies (2049 participants) showing modest improvements in attention and processing speed ( $g=0.158 ; 95 \% \mathrm{CI}, 0.055-0.260)$, executive function $(g=0.123 ; 95 \%$ CI, 0.021-0.225), and memory $(g=0.128 ; 95 \%$ CI, 0.015-0.241).

Depression is a common co-morbidity with a variety of cardiac conditions. Depression affects as many as $40 \%$ of patients with heart failure (HF), with up to $75 \%$ of patients reporting elevated depressive symptoms (Blumenthal et al., 2012a). For CHD, MDD affects $15-20 \%$ of cardiac patients and an additional $20 \%$ report elevated depressive symptoms (Blumenthal et al., 2012b). Blumenthal et al. (2012a) recently published an RCT of 2322 stable HF patients who underwent an aerobic exercise program (supervised for 1-3 months followed by home exercise for 9 months) or education and usual guideline-based HF care. Compared with usual care, aerobic exercise resulted in lower mean BDI-II scores at 3 and 12 months (differences of -0.76 and -0.68 , respectively). Another study by Blumenthal et al. (2012b) assessed efficacy of 4 months of aerobic exercise and antidepressant treatments (sertraline) in reducing depressive symptoms and improving cardiovascular biomarkers in depressed patients with CHD. At 4 months, exercise and sertraline were equally as effective at reducing depressive symptoms (HRSD) vs. placebo. Exercise tended to result in greater reductions in heart rate variability vs. sertraline.

When considering the anti-depressive effects of exercise - in addition to biological effects - we must consider psychosocial aspects. Studies have shown exercise regimens have a distraction effect (from negative thoughts and ruminations), provide a sense of mastery via the learning of new skills (Lepore, 1997), and hence enhance self-efficacy (Craft, 2005) and self-esteem (Salmon, 2001). A study by Craft (2005) found that those who experienced an increase in mood following exercise showed higher self-efficacy levels at 3 and 9 weeks post-exercise. Self-esteem is considered to be one of the strongest predictors of overall (Diener, 1984), subjective well-being and low self-esteem is considered to be closely related with mental illness (Fox, 2000). The abovementioned beneficial psychological effects may lead to the stress reducing and stressresilience enhancing effects of exercise (Salmon, 2001). Additionally, exercise regimens in a group setting may have a beneficial effect via training social skill deficits (Rimer et al., 2012).Therefore, considering the immunomodulatory effects of social support, i.e., social isolation stress is repeatedly shown to enhance inflammation in clinical and pre-clinical models (Hafner et al., 2011), the social interaction effects of PA interventions must be considered as a confounder.

Whilst the vast majority of research using PA in psychiatry is positive and encouraging, it is important to also consider potential pre-cautions during PA interventions. Some studies report no effect for PA in depression (Rimer et al., 2012). This may be explained by inappropriate intensity of PA, or a too short duration of PA as a treatment (Rimer et al., 2012). In order to enhance the potential for antidepressant effects, multiple authors now recommend exercise of moderate-intensity and of at least 8 weeks duration (Mead et al., 2008; Trivedi et al., 2011; Rimer et al., 2012). PA regimens must be tailored according to the individual patient's functional status and other co-morbidities. Failing to do so can lead to further morbidity and/or mortality. In patients with social phobia-related symptoms, the approach to PA interventions should be tailored appropriately.

\section{NEUROIMIMUNOLOGICAL EFFECTS OF PHYSICAL ACTIVITY IN DEPRESSION}

When considering the neuroimmunological effects of PA in depression, it is important to first outline the current understanding on neuroimmunological mechanisms of the depressionlike disease states. Therefore, the following section will outline these neuroimmunological mechanisms in detail; following, the neuroimmunological effects of PA will be examined. 


\section{NEUROIMMUNOLOGICAL CHANGES IN DEPRESSION}

The neuroimmunological changes found in depression involve humoral and cellular factors from both the innate and adaptive immune systems (Eyre and Baune, 2012c; Littrell, 2012). Humoral factors include PICs, AICs, C-reactive protein (CRP) as well as other immunomodulatory factors like CX3CL1, CD200, and IGF1 (Eyre and Baune, 2012b). Cellular factors include resident glia (e.g., astrocytes, microglia) and centrally migrating immune cells involved in protective immunosurveillance (e.g., CD4+ T cells and macrophages; Eyre and Baune, 2012b).

\section{Neuroinflammation and depression: a well recognized relationship}

The neuroinflammatory state is well known to be associated with the depressive phenotype (Dantzer et al., 2008; Dowlati et al., 2010). For example, a recent meta-analysis found a significant correlation between tumor necrosis factor (TNF- $\alpha$ ), IL-6, and CRP with depression in humans (Dowlati et al., 2010). Neuroinflammation is characterized by elevations in PICs and reductions in AICs and can arise within the CNS itself, or peripheral inflammatory signals can be transferred into the CNS (Dantzer et al., 2008; see Quan and Banks, 2007; for a review of peripheral-CNS pathways, including: the neural route, circumventricular organs, $\mathrm{BBB}$ transport of cytokines, and secretions from BBB cells). The neuroinflammatory state is known to cause neurovegetative or sickness-like symptoms, depression- and anxiety-like behaviors, as well as cognitive dysfunction and symptoms of Chronic Fatigue Syndrome (Dantzer et al., 2008; McAfoose and Baune, 2009; Dowlati et al., 2010; Miller, 2010; Yirmiya and Goshen, 2011; Bansal et al., 2012), and the causation of these phenotypic states by PICs has been modeled in both rodent and human models and extensively reviewed (Dantzer et al., 2008; Miller, 2010).

Neuroinflammation-based models of depression have shown PICs to impact on other major neurobiological systems involved in depression. Neuroinflammation affects the neurotransmitter systems by activation if the tryphophan degrading enzyme, indoleamine 2,3 dioxygenase (IDO), altering metabolism of tryptophan into neurotoxic metabolites (3-hydroxykyurenin, 3-HK and quinolinic acid, QA) and depleting its availability for serotonin (5-HT) synthesis (Miller, 2010; Dantzer et al., 2011; Moylan et al., 2012). Inflammation also stimulates the reuptake of monoamines from the synapse by increasing the activity and the density of 5-HT, noradrenaline, and dopamine transporters (Moron et al., 2003; Nakajima et al., 2004; Zhu et al., 2006). Evidence suggests these immune mechanisms adversely affected glutamatergic neurotransmission causing GLU to rise to neurotoxic levels (McNally et al., 2008; Hashimoto, 2009; Popoli et al., 2012). In the neuroinflammatory state PICs may disrupt the capacity of the glucocorticoid receptor to translocate to the nucleus where it normally acts to suppress the activity of pro-inflammatory transcription factors such as nuclear factor-kappa B (NF- $\mathrm{B})$ - this is termed glucocorticoid resistance (Dantzer et al., 2008; Miller, 2010; Muller et al., 2011). High levels of PICs impair processes of neuroplasticity in the HC, such as neurogenesis, LTP, neurotrophin production (e.g., brain-derived neurotrophic factor, BDNF), and synaptic plasticity (Miller, 2010; Eyre and Baune, 2012c). In the context of reduced neuroplasticity, elevations in neurotoxic oxidative stress products and markers of apoptosis are found in the HC (Moylan et al.,
2012). An in-depth assessment on the effects of inflammation on these systems is outside the scope of this review and have been outlined recently (see Dantzer et al., 2008, 2011; McAfoose and Baune, 2009; Muller et al., 2011; Moylan et al., 2012).

\section{Rationale for examining immune mechanisms in addition to inflammation}

Whilst the cytokine and neuroinflammatory models of depression have been helpful in understanding the neurobiology behind the depressive phenotype, there are a number of clinical and biological reasons for investigating neuroimmune mechanisms in addition to inflammation. These factors include:

- A recent meta-analysis by Hannestad et al. (2011) found results arguing against the notion that resolution of a depressive episode is associated with normalization of levels of circulating PICs. This analysis of 22 studies (603 subjects) found - when all antidepressants were grouped - these medications reduced levels if IL- $1 \beta$ with a marginal effect on IL-6 (using less stringent fixed-effects models); there was no effect on TNF- $\alpha$. However, a sub-group analysis of selective serotonin regulate inhibitors (SSRI) medication found a reduction in IL- 6 and TNF- $\alpha$. Other antidepressants did not reduce PIC levels.

- Recent evidence has emerged to suggest no effect or even an antagonistic effect for anti-inflammatory medications in depression. A large-scale prospective cohort study of treatmentresistant depression, the "sequenced treatment alternatives to relieve depression" (STAR $* \mathrm{D})$, found an antagonistic effect for anti-inflammatory compounds on ADs (Warner-Schmidt et al., 2011). Patients reporting concomitant non-steroidal anti-inflammatory drug (NSAID) or other analgesic treatment showed a reduced therapeutic response to citalopram, hence, the authors suggest concomitant use of NSAIDs may be an important reason for high SSRI treatment resistance rates (WarnerSchmidt et al., 2011). A recent re-analysis reached a similar conclusion, with more modest effects persisting after adjustment for potential confounding variables (Gallagher et al., 2012). Another recently published study shows no difference between infliximab, a TNF- $\alpha$ antagonist, and placebo in a recent 12 -week double-blind, placebo-controlled RCT for treatment-resistant depression (Raison et al., 2012). There was a significant effect for infliximab in individuals who had a high baseline hs-CRP ( $>5 \mathrm{mg} / \mathrm{L}$ ) and a significant effect for placebo-treated patients at a baseline hs-CRP of $<5$-mg/L. Schwartz and Shechter hypothesize anti-inflammatory drug compounds may block the production of brain-derived cytokines and chemokines which promote the migration of neuroprotective immune cells involved in protective immunosurveillance toward the CNS (Schwartz and Shechter, 2010b; Warner-Schmidt et al., 2011). Importantly, however, the use of NSAIDs may be most useful when used in the correct stage of neuroinflammatory diseases, i.e., administered early in the neuroinflammatory disease course when transmigratory immune cells have not come into effect (Schwartz and Shechter, 2010b).

- Evidence is emerging to suggest a neuroprotective and physiological role for "PICs." TNF- $\alpha$ and IL- 6 have been shown to play an integral roles in processes of memory and learning in 
both human and rodent studies, as well as having a physiological role in HC neuroplasticity (Carlson et al., 1999; Eyre and Baune, 2012c). The TNF- $\alpha$ gene ( $r s 1800629)$ is correlated with enhanced cognitive processing speed in a healthy human population (Baune et al., 2008a). The IL-6 gene ( $r$ s1800795) has been correlated with increased to HC volume in a healthy human population (Baune et al., 2012a). There are other studies outlining a neuroprotective effect of PICs in the brain (see below).

- From a clinical disease course perspective, there are other mechanisms in depression - in addition to inflammation - which may have a role in explaining the absence of correlation between the increase in neuroinflammation in aging and rates of depression. Since aging itself is related to higher levels of systemic inflammation and neuroinflammation (Hein and O'Banion, 2012), this should lead to higher rates of depression in old age, however, rates are highest in those aged 25-45 years, not in old age (Kessler et al., 2005). Other neuroimmune factors which may explain this scenario will be outlined below.

\section{DYSFUNCTION OF NEUROPROTECTIVE IMMUNE FACTORS IN DEPRESSION}

When considering neuroimmunological factors in depression, historically the focus has mainly been on high levels of PICs and their detrimental effects on the brain. However, research is beginning to suggest a significant role for neuroprotective neuroimmune factors in depression and other neurobiological disorders (e.g., multiple sclerosis and AD; Martino et al., 2011; Kokaia et al., 2012). When considering these neuroprotective factors in depression, their loss of function may exacerbate the depression-like behaviors (Schwartz and Shechter, 2010b). The following section will outline evidence suggesting a possible beneficial role for a variety of neuroimmune factors.

\section{Neuroprotective and physiological effects of cytokines}

There are a number of cytokines are found to have neuroprotective and physiological effects.

Interleukin- 6 has been found to have neuroprotective effects via gp130 signaling and related pathways [i.e., Janus Kinase (JAK)/Signal Transducer and Activator of Transcription (STAT), Mitogen-activated Protein Kinase (MAPK)/cAMP Response Element-binding (CREB), Ras-MAPK, Phosphatidylinositol 3kinases (PI3K); Baune et al., 2012a]. These mechanisms affect the production of neurotrophic factors, cellular survival, and apoptosis (Baune et al., 2012a). A recent imaging genetics study investigated the association between the $I L-6$ gene and brain morphology in a large cohort of healthy adult participants in a whole-brain analysis approach (Baune et al., 2012a). Carriers of the G-allele of the IL-6 genetic variant $r s 1800795(-174$ $C / G$ ) showed a significant association with larger HC volumes on the right side in healthy subjects. This genotype effect was remarkably specific to the $\mathrm{HC}$, with no other structure surviving statistical threshold for the entire brain. The findings are suggestive of a neuroprotective role of the $I L-6$ gene [ $r s 1800795$ $(-174 C / G)]$ on HC morphology. Supporting a role of IL-6 in neuroproliferation is an in vivo study showing that IL-6 knockout mice have reduced proliferating NSCs specifically in the HC, hence underlining the importance of IL- 6 in cell proliferation and cell survival (Bowen et al., 2011). However, other similar studies have shown no effect or a negative effect for IL-6 in neurogenesis processes (Eyre and Baune, 2012c). The difference between the pro- and anti-neurogenic effects of IL-6 may reflect differences in amounts and conditions used experimentally (Eyre and Baune, 2012c).

Tumor necrosis factor- $\alpha$ is thought to exert its protective and restorative effects primarily via TNFR2 ( 775 ; primarily neuroprotective and neuroregenerative pathway) and related signaling pathways [i.e., IкB kinase (IKK)/Nuclear Factor $\kappa \mathrm{B}(\mathrm{NF}-\mathrm{kB})$, Transforming Growth factor $\beta$-activated Kinase 1 (TAK-1), PI3KPKB-Akt, c-Jun N-terminal kinases (JNK), and IL-6), as opposed to the TNFR1 (p55; primarily neurodegenerative; Eyre and Baune, 2012a; Santello and Volterra, 2012). Importantly, whether the outcome of TNF- $\alpha$ signaling is protective or damaging may depend upon duration of NF-кB activation (Santello and Volterra, 2012). TNF- $\alpha$ has been found to exert beneficial effects in depressionrelated processes, e.g., cognitive function and $\mathrm{HC}$ neurogenesis (Eyre and Baune, 2012a; Santello and Volterra, 2012). During relatively health aging processes, it has been shown that the TNF- $\alpha$ gene ( $r$ 1800629) has protective effects on cognitive processing speed (Baune et al., 2008a) and has been associated with cognitive processes (e.g., response inhibition, error processing, attentional processes, and mental rotation) in young health individuals (Eyre and Baune, 2012a). In behavioral studies, TNF- $\alpha$ deficient mice exhibit impaired HC-dependent memory function in the Morris Water Maze suggesting that during early stages of brain development basal levels of TNF is required for memory and learning (Baune et al., 2008b).

Interleukin- 4 has been found to have a beneficial role in depression-like behaviors and a neuroprotective effect. The release of IL-4 from CNS-specific autoreactive CD4+ T cells involved in protective immunosurveillance - in response to increased neurotoxicity (Ron-Harel et al., 2011) - binds to IL-4 receptor on the cytotoxic microglia (Kipnis et al., 2008) causing downregulation of PIC production, induction of BDNF and IGF-1, and an elevation in neurogenesis (Butovsky et al., 2005, 2006b; Lyons et al., 2009; Martino et al., 2011). Microglia under quiescent conditions, after exposure to IL-4 or low levels of IFN- $\gamma$ (Butovsky et al., 2006b), have been shown to support neurogenesis and NSC differentiation and migration in vitro (Aarum et al., 2003; Butovsky et al., 2006b; Walton et al., 2006). IL-4 is also shown to promote the creation of neuroprotective M2-type microglial phenotype (Godbout et al., 2012). A recent study found central IL-4 administration increased microglial-specific M2a-type genes including Arginase, $I L-1 R \alpha$, and BDNF (Godbout et al., 2012). Microglia activated by IL-4 remain committed to their protective phenotype (M2-type) even when exposed to a threatening environment in the form of LPS, and, exposure of microglia, pre-activated to a cytotoxic phenotype, to IL-4 induces a phenotype switch toward neuroprotection (Butovsky et al., 2005; Schwartz et al., 2006). A study rodent by Derecki et al. (2010) shows T cell-derived IL-4 to have beneficial effects on the regulation of cognitive function in rodents via meningeal myeloid cell phenotypes producing BDNF. IL-4 knockout mice show greater sickness behavior (measured by exploratory behavior) than wildtype mice exposed to LPS (Lyons et al., 2009). Interestingly, Kim et al. (2011) proposes T-bet deficient mice may 
have a neuroprotective effect by creating a predominance of Th2derived IL-4, which may in turn stimulate meningeal myeloid cell BDNF production. T-bet is a Th1-specific T-box transcription factor which regulates CD4+ Th1 development by inducing endogenous Th1 cytokines, while simultaneously repressing Th2 development (Wong et al., 2008).

A role for IL-10 in neuroprotection and the prevention of depression-like behavior has been suggested. Central administration of IL-10 prevents the emergence of behavioral signs of depression in an LPS model of sickness behavior (Bluthe et al., 1999). IL-10 over-expression mice display less anxiety-like behaviors, while IL-10 knock-out rodents display greater anxiety and depression-like behavior (forced-swim test) with these effects more pronounced in females (Mesquita et al., 2008). In human studies, IL-10 is found to be reduced in the depressed state (Himmerich et al., 2010). Further papers examining the neuroprotective effects of IL-10 can be found in Raison and Miller (2011).

\section{Immunomodulatory factors}

Insulin-like growth factor-1 is a major neurotrophic factor involved in neuroplastic functions such as neurogenesis and is critical in normal memory and LTP functions (Trejo et al., 2007). Recent evidence suggests IGF-1 also has added immunomodulatory effects (Park et al., 2011a,b). In an LPS model of depression, central administration of IGF-1 is shown to prevent LPS-induced sickness- and depression-like behavior (Park et al., 2011a,b) in association with an induction of BDNF and a reduction of TNF- $\alpha$, IL-1 $\beta$, and iNOS in the pre-frontal cortex (PFC; Park et al., 2011b). Given the levels of IGF-1 have been found to be low in rodent models of depression (Mitschelen et al., 2011), the absence of this anti-inflammatory factor may exacerbate the neuroinflammatory and anti-neuroplastic state in depression.

CX3CL1 is a chemokine expressed by healthy neurons which has its receptor, CX3CR1, in membrane bound form or as soluble ligand (Rogers et al., 2011). It has an important role in inhibiting the activation of microglia (Rogers et al., 2011). A recent study with CX3CR1 knock-out mice and the LPS model of sickness behavior found a deficiency in the action of CX3CL1 resulted in protracted microglial activation, as measured by IL- $1 \beta$ and CD14 (Corona et al., 2010). These mice have extended LPSinduced depression-like behavior in association with the activated microglial phenotype described (Corona et al., 2010). In another study with CX3CR1 knock-out mice, a lack of the CX3CR1 receptor resulted in contextual fear conditioning (associative memory) and Morris Water Maze deficits (spatial memory), as well as impairment in LTP (Rogers et al., 2011). Disruption of the CX3CL1/CX3CR1-pathway in young rodents decreases both survival and proliferation of HC neural progenitor cells (Bachstetter et al., 2011).

CD200 is a membrane glycoprotein which has been identified as an immune-suppressive molecule (Cox et al., 2012). It is expressed in neurons and oligodendrocytes, but not on microglia (Cox et al., 2012). The receptor for CD200, CD200R, is also a membrane glycoprotein and is primarily restricted to cells of the myeloid lineage, hence being found on microglia, but not neurons or astrocytes (Cox et al., 2012). The interaction between CD200 and its receptor play a significant role in maintaining microglia in a quiescent state, therefore, a decrease in CD200 expression is associated with evidence of microglia activation (Cox et al., 2012). A rodent study by Frank et al. (2007) shows an inescapable shock model of stress over $24 \mathrm{~h}$ resulted in a downregulation of HC CD200 in association with enhanced LPS-induced cytokine production in $\mathrm{HC}$ microglia. This suggests stress can activate microglia via downregulation of CD200, enhancing the PIC production of microglia (Frank et al., 2007). A study by Cox et al. (2012) found a CD200 fusion protein (CD200Fc), activator of CD200R, attenuated age-related microglial immunoreactivity in the HC (indicated by MHCII, CD40, and iNOS). CD200Fc also attenuated LPS-induced microglial activation (indicated by elevated MHCII, CD40, CD11b, and CD68) and LTP deficits (Cox et al., 2012). Using CD200 knock-out mice and LPS-induced sickness behavior, Costello et al. (2011) found the neuroinflammatory changes resulting from CD200 deficiency have a negative impact on LTP in the CA1 region of the dentate gyrus. Interestingly, a study by Lyons et al. (2009) has shown IL-4 as a key inducer of CD200 expression.

\section{Dysfunction of protective immunosurveillance}

Emerging data suggests a role for CNS-specific autoreactive CD4+ T cells, blood-derived macrophages (in the form of M2 alternatively activated macrophages) in physiological, protective immunosurveillance functions of the brain (Derecki et al., 2010, 2011; Martino et al., 2011; Ron-Harel et al., 2011). Evidence suggests these cell types may have established a physiological connection between the immune system and the brain, and have assisted in explaining processes of HC-dependent neurogenesis and cognitive dysfunction (Kipnis et al., 2004b; Butovsky et al., 2006b, 2007; Ziv et al., 2006; Brynskikh et al., 2008; Derecki et al., 2010, 2011), anxiety- and depression-like behavior (Cohen et al., 2006; Lewitus et al., 2008; Cardon et al., 2010) due to an insufficient immune response (Derecki et al., 2010, 2011; Schwartz and Shechter, 2010a,b; Ron-Harel et al., 2011). The role of these cells in neuroprotection and higher neurocognitive functions has been reviewed in detail elsewhere (Martino et al., 2011; Yirmiya and Goshen, 2011); however, a brief summary will be given, below.

Immune cells involved in protective immunosurveillance can populate meningeal areas of the choroid plexus and the cerebrospinal fluid, hence gaining access to the healthy brain without entering the parenchyma (Ransohoff et al., 2003; Derecki et al., 2010, 2011; Schwartz and Shechter, 2010a). CNS-specific autoreactive CD4+ T cells are suggested to react to three signals, (1) T-cell receptor (TCR; Ron-Harel et al., 2011), (2) co-stimulatory signals (CD28/CD80,86; Jenkins and Johnson, 1993), and (3) PICs and reactive oxygen species (ROS; Curtsinger et al., 1999; Tse et al., 2007; Ron-Harel et al., 2011). The T cells in question, activated in response to increased neurotoxicity (Ron-Harel et al., 2011), are thought to secrete increased levels of IL-4 (Ron-Harel et al., 2011), where IL-4 penetrates the brain parenchyma and binds to IL-4R on the cytotoxic microglia (Kipnis et al., 2008). Exposure of cytotoxic microglia to IL-4 causes downregulation of PIC secretion, induction of BDNF and IGF-1 secretion, and an elevation in neurogenesis (Butovsky et al., 2005, 2006b; Martino et al., 2011). All of these signals support the restoration of brain homeostasis (Ron-Harel et al., 2011). Furthermore, the T cells boost infiltration 
of neuroprotective blood-borne monocytes upon need (Shechter et al., 2009). A recent commentary by Ron-Harel et al. (2011) suggests any destabilization in brain homeostasis that cannot be locally contained by microglia and/or astrocytes will increase $\mathrm{T}$ cell recruitment as well as subsequent IL-4 release and recruitment of blood-derived macrophages.

According to the "protective immunosurveillance" model, increased susceptibility to mental illness may result from a deficiency in circulating T cells and the IL-4 they can produce, as the IL-4 mediates processes which are able to counteract neuroinflammation and restore brain homeostasis (Ron-Harel et al., 2011). Indeed, the brains of immune-deficient mice show accumulation of toxicity (i.e., increased glyoxalase-1, a compensatory mechanism against free radical and carbonyl levels; Ron-Harel et al., 2011).

According to the protective immunosurveillance model, activation of CNS-specific autoreactive CD4+ T cells (mentioned above) support the infiltration of neuroprotective, alternatively activated M2 macrophages to the sub-arachnoid meningeal spaces and choroid plexus, via IL-4 and IFN- $\gamma$ secretion (Derecki et al., 2010, 2011; Ron-Harel et al., 2011). These infiltrating macrophages, together with the microglia they regulate, remove dead cells and cellular debris, buffer toxic compounds (such as GLU and ROS), and produce growth factors (i.e., BDNF and IGF-1), while downregulating inflammation-associated compounds such as IL- $1 \beta$, TNF- $\alpha$, iNOS, and COX-2 (Hauben et al., 2000; Butovsky et al., 2005, 2006a,b, 2007; Shaked et al., 2005; Beers et al., 2008; Chiu et al., 2008; Rolls et al., 2008; Shimizu et al., 2008; KoronyoHamaoui et al., 2009; Shechter et al., 2009; Derecki et al., 2010, 2011; Prinz et al., 2011). These neurobiological functions are thought to contribute to blood-derived macrophages support of learning and memory (as determined via the Morris Water Maze and Barnes Maze; Derecki et al., 2010, 2011). Importantly, intravenous injection of M2 cells into immune-deficient mice can circumvent the need for CNS-specific autoreactive CD4+ T cells (Derecki et al., 2011). For a review of the role of blood-derived macrophages see recent papers (Derecki et al., 2010; Martino et al., 2011; Yirmiya and Goshen, 2011).

The type of macrophage - classical (M1), alternatively activated (M2), and deactivated types - determines the role in sickness behavior (for thorough review see Moon et al., 2011). Classical macrophages produce PICs and, hence, induce sickness behaviors (Dantzer et al., 2008; Moon et al., 2011). M2 macrophages which reduce PIC production, as outlined above, are associated with a reduction in sickness behavior (Derecki et al., 2010, 2011; Sherry et al., 2010). Deactivated macrophages which inhibit PIC production via IL-10 secretion are also thought to have beneficial effects of sickness behaviors, however, this finding has not been replicated (Moon et al., 2011).

It is important to mention a recent critique of the protective immunosurveillance concept recently produced by Rook et al. (2011). One important issue raised is that the phenotype of the neuroprotective, CNS-specific autoreactive CD4+ T cells is poorly understood (Rook et al., 2011). The authors suggest immune cells involved with the function of protective autoimmunity is likely from a regulatory cell - not always CD25+ - given the involvement of IL-4 and IL-10. Suggestions for potential cell types include
Th3, Tr1, Th2, IL-10+ ${ }^{+}$H1, CD8+ reg cells, regulatory Foxp3+ NKT, IL- $10^{+}$CD56 $6^{\text {bright }} \mathrm{NK}$, or various other IL-10-secreting cell types (Fujio et al., 2010; Rook et al., 2011). Another important consideration raised is the effect of T cell produced IL- 4 on T reg differentiation. $\mathrm{T}$ cell differentiation into the $\mathrm{T}$ reg cell type can be enhanced or opposed by IL-4 depending on the context (Chapoval et al., 2010; Rook et al., 2011). Further, one study shows IL-4 increased certain chemokines (CCL1, CCL17, and CCL22) in an experimental autoimmune encephalitis (EAE) model capable of recruiting $\mathrm{T}$ regs (Butti et al., 2008). The above mentioned issues are relevant to the neuroimmune model of depression considering the dynamic relationship between $\mathrm{T}$ regs and effector $\mathrm{T}$ cells.

\section{Glial cells}

The role of the immunocompetent glia, astrocytes, and microglia, in depression is complex and poorly understood (Beumer et al., 2012); importantly, however, there is a developing literature supporting a neuroprotective effect of these cells under certain conditions (Schwarz and Bilbo, 2011, 2012; Ekdahl, 2012). The follow section will summarize most recent evidence available in this field.

\section{Microglia}

The function of microglia is dynamic even in the resting state whereby they continually survey their microenvironments by extending and contracting processes into nearly synapses (Bilbo et al., 2012). Microglia are the resident macrophages of the CNS and are recognized as the primary component of the neuroimmune system (Ekdahl, 2012). Once activated - by chronic stress conditions, or immune challenge with LPS or PICs - microglia are capable of producing PICs and neurotoxic mediators such as nitric oxide, PGE2, and superoxide anions (Liu et al., 2011; Bilbo et al., 2012; Ekdahl, 2012). A recent study by Walker and colleagues has shown a role for microglia in mediating the effects of stress on PFC neuronal function and PFC-regulated behavior (Hinwood et al., 2012). This study found restraint stress conditions caused a decline in working memory performance associated with increased microglial activity (measured by a $25 \%$ increase in Iba- 1 labeling, $\triangle$ FosB, and a hyper-ramified state) in the medial PFC and no association was found with increased antigen presentation (MHCII) or apoptosis (caspase-3; Walker et al., 2011).

Given the pre-existing association with the inflammatory hypothesis of depression much research centers on reducing the PIC production of microglia (Liu et al., 2011). Recent evidence suggests a neuroprotective function of microglia under certain circumstances (Yirmiya and Goshen, 2011; Ekdahl, 2012). For example, microglia under quiescent conditions, after exposure to IL-4 or low levels of IFN- $\gamma$ (Butovsky et al., 2006b), have been shown to support neurogenesis and NSC differentiation and migration in vitro (Aarum et al., 2003; Butovsky et al., 2006b; Walton et al., 2006). Microglia activated by IL-4 remain committed to their protective phenotype even when exposed to a threatening environment in the form of LPS, and, exposure of microglia preactivated to a cytotoxic phenotype to IL-4 induces a phenotype switch toward neuroprotection (Butovsky et al., 2005; Schwartz et al., 2006). Exposure of rats to environmental enrichment (EE) increases neurogenesis alongside increased HC microglia proliferation (microglia assumed a neuroprotective phenotype expressing 
MHC II and IGF-1; Ziv et al., 2006). As mentioned previously, the pro-neurogenic effects of microglia may be related to their interactions with CNS-specific autoreactive CD4+ T cells, this was further confirmed by a study showing transgenic mice with an excess of these $\mathrm{T}$ cells - and associated increases in neurogenesis - showed attenuated neurogenesis by chronic treatment with the microglial inhibitor, minocycline (Ziv et al., 2006). Furthermore, a rodent model of amyotrophic lateral sclerosis (ALS) illustrates the interaction between $\mathrm{T}$ cells and microglia whereby Th1 cytokines promote M1 microglia and Th2 or Treg cytokines promote M2 microglia (Chiu et al., 2008). Microglia were also shown to support neurogenesis in adrenalectomized rodents via TGF- $\beta$ (Battista et al., 2006; Mathieu et al., 2010). Opposing the above neuroprotective findings is a rodent study demonstrating that PA-induced neurogenesis was not associated with microglial proliferation or activation, and no indication of T-cell-microglial interactions (i.e., no MHC II expression or T cells in the HC; Olah et al., 2009).

In summary, microglial function is closely intertwined with the immune system and neurogenesis (Ekdahl, 2012), with the crosstalk between these systems requiring further investigation. For instance, a recent review by Ekdahl (2012) suggests microglial activation patterns may by region-specific. Moreover, there appears to be a primarily beneficial interaction between microglia and new neurons in the intact brain, however, the cross-talk is complex and probably double-edged in pathological conditions, especially following long-term microglial activation (Ekdahl, 2012).

\section{Astrocytes}

Astrocytes are physically and functionally appositioned with most synapses, known as the "tripartite synapse" (Araque et al., 1999). They possess immune-like properties whereby they have an ability to respond to inflammatory cytokines (particularly IL-1 $\beta$ ), to secrete PICs (i.e., TNF- $\alpha$ and IL-6) and to phagocytose cellular processes and debris (Yirmiya and Goshen, 2011). These cells play an important role in neural and synaptic functioning. For example, a rodent study by Bracchi-Ricard et al. (2008) shows female mice where the transcription factor NF- $\kappa \mathrm{B}$ was inhibited specifically in astrocytes displayed deficits in learning, memory, and LTP. These cells were also found to mediate homeostatic synaptic scaling following prolonged inhibition of neuronal activity via TNF- $\alpha$ secretion, a known synaptic strength enhancer (Stellwagen and Malenka, 2006; Kaneko et al., 2008). The role of astrocytic IL-1 signaling in memory functioning and LTP was recently demonstrated by Ben Menachem-Zidon et al. (2011). In this study neural precursor cells (NPCs) derived from either WT or IL-1rKO neonatal mice were labeled with BrdU and transplanted into the $\mathrm{HC}$ of either IL-1rKO or WT adult host mice. Transplanted NPCs showed long-term survival and differentiated into astrocytes (expressing GFAP and S100 $\beta$ ), but did not differentiate into neurons. Several weeks post-transplantation, IL-1rKO mice transplanted with IL-1rKO cells, or sham operated, displayed severe memory disturbances and a marked impairment in LTP. However, IL-1rKO mice transplanted with WT NPCs (expressing IL-1R) displayed complete rescue of the impaired memory functioning, as well as partial restoration of LTP. IL-4 is also found to be important in astrocyte functioning with the secretion of BDNF by in vitro astrocytes being markedly enhanced by this cytokine (Martino et al., 2011). Furthermore - and in fitting with the abovementioned model of protective immunosurveillance by Schwartz et al. - astrocytes also acquire a neuroprotective phenotype following their co-culture with T cells (Garg et al., 2008).

There is a paucity of evidence correlating the role of the abovementioned glial cells in models and tests of depression-like behavior. This is an important area for future research as these cells appear to be involved in depression-related pathophysiological processes.

\section{Additional cellular immune factors}

The role of T regs in depression is uncertain, and may be both positive and negative in depression pathophysiology depending on the surrounding environment (Cohen et al., 2006; Himmerich et al., 2010). In relation to the positive effects of $\mathrm{T}$ regs, some authors propose these cells may function to inhibit inappropriate or excessive immune responses, i.e., PIC production (Dantzer et al., 2008; Miller, 2010). Some human studies have found reduced IL-10 and TGF- $\beta$ have been found in depressed patients, and are thought to be consistent with reduced $\mathrm{T}$ reg expression and/or function (Myint et al., 2005; Sutcigil et al., 2007; Dhabhar et al., 2009; Musil et al., 2011). One study found decreased $\mathrm{T}$ regs, alongside intracellular Foxp3, in association with IL-10 and TGF- $\beta$ in depressed patients vs. controls (Li et al., 2010). A second study found 6 weeks of $\mathrm{AD}$ treatment led to increased $\mathrm{T}$ reg $\left(\mathrm{CD} 4+\mathrm{CD} 25^{\mathrm{hi}}\right)$ percentage in association with decreased IL-1 $\beta$ (Himmerich et al., 2010). A recent rodent study shows $\mathrm{T}$ reg cell depleted mice undergoing chronic immobilization stress displayed markedly increased anxiety in the Elevated Plus Maze and increased depression-like behavior in the Forced-Swim Test (Kim et al., 2012). These finds were found in correlation with elevated serum cytokines (i.e., IL6 , TNF- $\alpha$, IL-2, IFN- $\gamma$, and IL-4) and reduced levels of HC 5-HT. In addition, a rodent model of cholestatic liver disease due to bile duct ligation found $\mathrm{T}$ regs suppress sickness-like behavior alongside inhibiting monocyte and hepatic IL-6 production, and subsequent signaling via circulating IL-6 acting (via p-STAT3 at the level of the cerebral endothelium; Nguyen et al., 2012). However, $\mathrm{T}$ regs have also been found to inhibit the beneficial effects of CNS-specific autoreactive CD4+ T cells on mitigating stressinduced anxiety-like behaviors in rodents (Cohen et al., 2006). This suggests $\mathrm{T}$ regs may inhibit the neuroprotective functions of these autoreactive $\mathrm{T}$ cells, a counterproductive effect. Interestingly, other studies with an optic nerve injury model have shown both Treg-free CD4+ T cells and T regs, respectively, can exhibit neuroprotective functions via preventing neuronal cell loss (Kipnis et al., 2004a). T regs exhibit significant plasticity and can lose regulatory activity, expressing effector cell function under certain circumstances (Zhou et al., 2009). Therefore, the balance of these two cells types may play a role in neuroprotective functions. Interestingly, $\mathrm{T}$ regs constitutively express CD25, a high affinity IL-2 receptor. The expression of CD25 is thought to be one of the ways by which $\mathrm{T}$ regs suppress proliferation of $\mathrm{T}$ effector cells, that is, by acting as a sink for IL-2 which is needed for T effector cell proliferation (Walsh and Kipnis, 2011). Interestingly, IL-2 is known to increase the suppressive abilities of $\mathrm{T}$ regs (Kohm et al., 2006), hence, the reduction of IL-2 which is seen in some studies of depression may 
reduce the anti-inflammatory effects of $\mathrm{T}$ regs (Anisman et al., 1999; Blume et al., 2011). A recent review paper summarizes literature suggesting $\mathrm{T}$ reg phenotypes are flexible depending on background chemokine and cytokine levels (Rook et al., 2011). Flexibility of phenotype means these cells can change from antito pro-inflammatory functions (Rook et al., 2011); indeed, authors remark that T-cell phenotype may change from the start to the end of studies (Rook et al., 2011). Furthermore, gut microbiota may affect the immunosuppressive function of $\mathrm{T}$ regs as well as their effects on higher neurocognitive behaviors of the brain (Rook et al., 2011). Clearly, the effect of $\mathrm{T}$ regs in depression requires further research.

\section{Other T-cell subtypes in depression - Th1, Th2 cells, and T-bet}

The balance of Th1 vs. Th2 cytokines in depression is currently debated by prominent authors in the field (Capuron and Miller, 2011; Rook et al., 2011). The majority of evidence suggests a net Th1 production as a key feature of immune dysfunction in depression, however, some studies suggest increased Th2 production (Myint et al., 2005; Capuron and Miller, 2011; Rook et al., 2011; Leonard and Maes, 2012). Th1 cells can produce IFN- $\gamma$, IL2 , and TNF- $\alpha$; Th 2 cells can produce IL-4, IL-6, and IL-10. Recent evidence suggests T-bet is associated with depression-like behaviors (Wong et al., 2008; Kim et al., 2011). T-bet deficient mice, Th1/IFN- $\gamma$ depleted, are shown to be resistant to stress-induced depression-like behavior and stress-induced neuroinflammation (i.e., IL-6 and TNF- $\alpha$; Kim et al., 2011). A clinical study by Wong et al. (2008) in a sample of Mexican Americans with major depression, shows evidence that single nucleotide polymorphisms (SNPs) in the T-bet (Tbx21) gene, which is critical for helper T (Th) 1-cell function, are associated with susceptibility to major depression. Moreover, the same study showed T-cell involvement in AD treatment response of genes associated with T-cell development (T-cell antigen receptor- $\varepsilon$ subunit of T3, CD3E; Wong et al., 2008).

\section{BALANCING BENEFICIAL AND DETRIMENTAL EFFECTS OF THE NEUROIMMUNE SYSTEM IN DEPRESSION}

In the sections above we have outlined both the beneficial and detrimental effects of the neuroimmune system in depression. From this information, we suggest that depression-related pathophysiology and depression-like behaviors may be dictated by the balance between the beneficial and detrimental effects of neuroimmune factors. See Figure 1 for a graphical representation of this balance. It is possible that when the balance is skewed toward the detrimental effects of the neuroimmune system, this leads to the development of depression-like behaviors, may prolong depressive episodes and lead to more severe symptomatology and behaviors. Alternatively, if the balance becomes skewed toward the beneficial effects of the neuroimmune system, this would reduce symptomatology and behavior and may drive the end of depressive episodes and prolong relapse remission.

\section{NEUROBIOLOGICAL EFFECTS OF PHYSICAL ACTIVITY IN DEPRESSION}

The neurobiological effects of PA in depression include effects on neurotransmitter, neuroendocrine systems, effects on neuroplasticity, and effects on neuroimmunological factors. The following section will outline the effects of PA on these systems, below, with a focus on neuroimmunological factors.

\section{Neurobiological effects}

Physical activity has been shown to upregulate monoamine neurotransmitters in the brain (e.g., 5-HT, dopamine, and noradrenaline) as well as endorphins (Knochel et al., 2012; Lautenschlager et al., 2012; Sarris et al., 2012). Reductions in glucocorticoid stress hormones are also found alter PA interventions whereby PA appears to re-regulate the HPA axis (Eyre and Baune, 2012c). Oxidative stress is reduced in the hippocampus in pre-clinical populations (Marosi et al., 2012).

Hippocampal neuroplasticity (e.g., neurogenesis, HC volume, and neurotrophin production) is increased with PA interventions in both clinical and pre-clinical populations (Erickson et al., 2012; Knochel et al., 2012; Lautenschlager et al., 2012). Pereira et al. (2007) reported that aerobic exercise resulted in increased HC blood volume which correlated with improved aerobic capacity and neurogenesis in the dentate gyrus. A recent RCT by Erickson et al. (2011) found that an aerobic exercise program in older adults, for 3 days a week over 1 year, increased HC volume by $2 \%$. This was associated with increased serum BDNF and improvements in spatial memory. Further work is required to investigate the effects of PA on neuroplasticity in the PFC and amygdala.

\section{Neuroimmunological effects in clinical populations}

A recent study by Rethorst et al. (2012) aimed to determine the extent to which inflammatory markers can be used to predict treatment response to exercise treatment, and if this effect was dependent upon the dose of exercise. This prospective study used participants who were incomplete responders to an SSRI and randomized them to two doses of aerobic exercise for 12 weeks [ 4 or 16 kilocalories per kilogram of body weight per week (KKW))] $16 \mathrm{KKW}$ was designed to meet or exceed current PA guidelines for public health from professional associations. The study found participants with a high baseline TNF- $\alpha(>5.493 \mathrm{pg} / \mathrm{ml})$ had a greater reduction in depressive symptoms (measured by IDS-C) than those with a low TNF- $\alpha$ level. Interestingly, this finding may suggest TNF- $\alpha$ as a moderator between SSRI and exercise treatment, and TNF- $\alpha$ levels could be used to recommend exercise rather than medication as part of a personalized treatment algorithm (Rethorst et al., 2012). This is given Eller et al. (2008) found high baseline TNF- $\alpha$ associated with non-response to an SSRI, and the Hannestad et al. (2011) meta-analysis also supports this association. There was a significant correlation between change in IL-1 $\beta$ and depression symptoms for the 16 KKW group, but not the 4 KKW group. The meta-analysis by Hannestad et al. (2011) also found a reduction in IL- $1 \beta$ correlated with better outcomes with SSRIs. Interestingly there was no change in cytokines levels following either exercise dosage. The authors suggest this may have occurred due to pre-treatment with SSRIs - a well known antiinflammatory agent (Hannestad et al., 2011) - which obscured the ability to detect changes in cytokine levels. Indeed, many past studies have shown exercise to have a robust anti-inflammatory effect in both human and rodent studies (Rethorst et al., 2011; Eyre and Baune, 2012a).

Another recent study by Irwin and Olmstead (2012) utilized a 9-week TCC program in a healthy older adult population to investigate the effect of exercise on depression symptoms. This study 


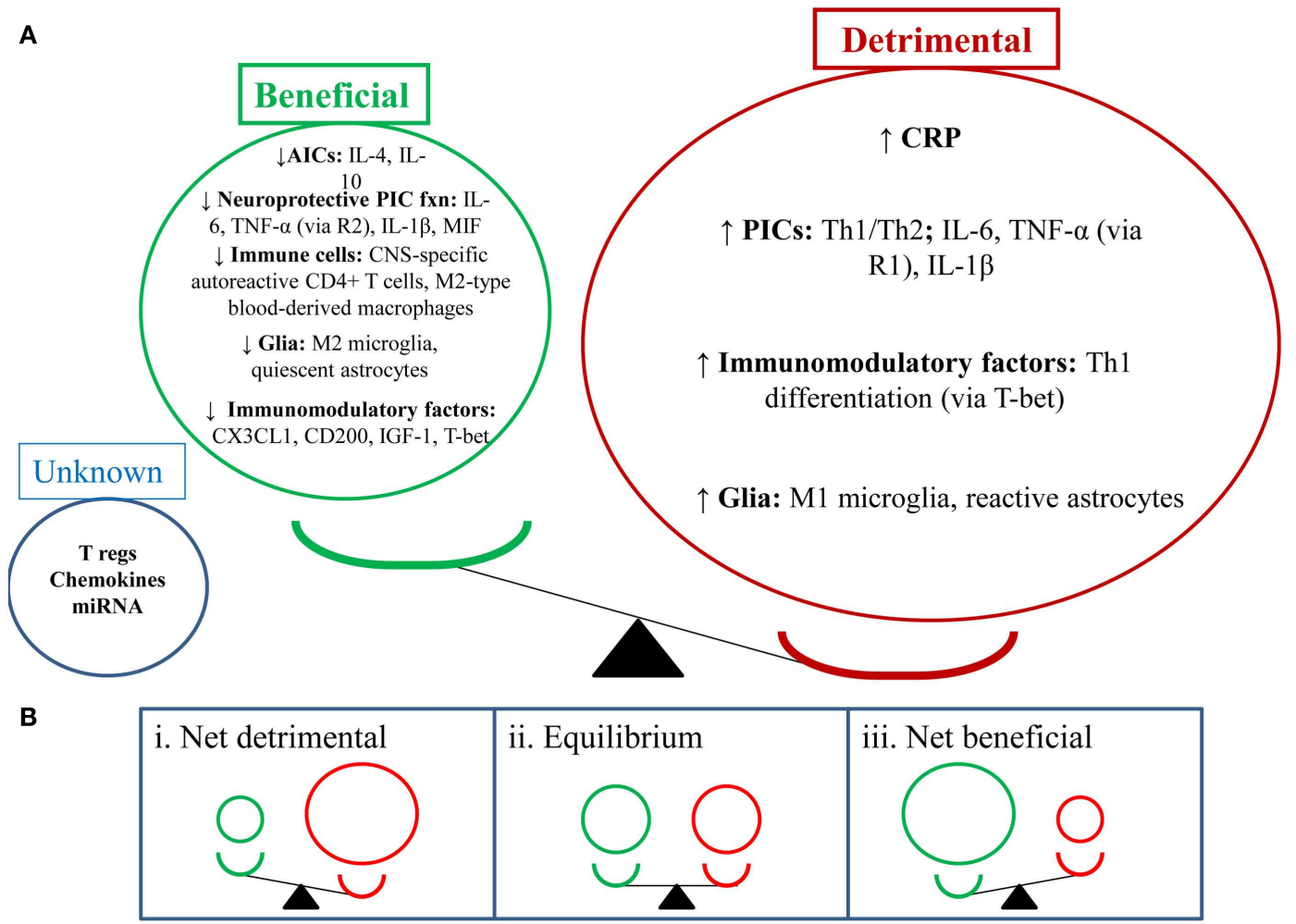

FIGURE 1 | Depression-like behavior: balancing the beneficial and detrimental effects of the neuroimmune system. (A) This section shows the balance of the detrimental (red) and beneficial (green) effects of neuroimmune factors in the depressed state (i.e., detrimental factors out way beneficial factors). NB: depression-like behavior includes sickness-like behavior, anhedonia, anxiety-, and cognition-like behaviors. (B) This section shows a number of potential outcomes for the balance between the abovementioned neuroimmune factors. (i) Shows a net detrimental effect which would lead to depression-like behaviors; clinically this could mean a depressive episode and could also increase relapse rates. (ii) Shows an equilibrium position which may suggest a stable/steady state in behavior; clinically this could mean a euthymic state. (iii) Shows a net beneficial effect which may attenuate depression-like behavior; clinically this could mean reduction or resolution of depressive symptoms and reduced relapse rates. found TCC reduced depressive symptoms (BDI) in correlation with a reduction in IL-6 levels. TCC, however, had no effect on cellular markers of inflammation (i.e., sIL-1ra, sIL-6, sICAM, and IL-18). The authors suggest PA treatments may modulate IL-6 via decreasing sympathetic outflow. Aging and stress are associated with increases in circulating catecholamine levels, which are known to increase IL-6.

A study by Kohut et al. (2006) found aerobic exercise reduced pro-inflammatory factors (i.e., CRP, IL-5, TNF- $\alpha$, and IL-18) more than a combination of flexibility and strength exercise over a 10 -month period. These exercise types both reduced depressive symptoms in the Geriatric Depression Scale (GDS).

The robust lipolytic effects of PA are suggested to play a role in the antidepressant effects of PA in depression, via reducing the systemic pro-inflammatory state seen in obesity (Gleeson et al., 2011). A high visceral fat mass has been shown to cause a chronic inflammatory state, and this chronic inflammatory state may link depression and obesity (Stuart and Baune, 2012). Gleeson et al. (2011) also suggests physical inactivity is a risk factor for the accumulation of visceral fat which may predispose individuals to chronic illness like depression and heart disease via systemic PIC production by visceral fat mass.
See Tables 1 and 2 for clinical studies examining the effects of exercise on neuroimmunological factors with and without depressive symptom correlations, respectively.

\section{Neuroimmunological effects in pre-clinical populations}

As seen in Tables 3 and 4, there are a large number of studies investigating the neuroimmunological effects of PA. Studies have been variously conducted with and without behavioral correlates. The following section will summarize the salient studies in this field.

A recent study found a voluntary exercise regimen to be associated with increased HC MIF, as well as Bdnfand Tph2 (tryphophan hydroxylase, involved in the synthesis of 5-HT) gene expression (Moon et al., 2012). These changes occurred in the context of reduced depression-like behavior (FST), and the effect of PA on these factors was mediated by the CD74-GTPase (MIF receptor) and RhoA-ERK1/2 pathway. MIF is a PIC expressed in the CNS whose deletion is associated with increased anxiety- and depression-like behaviors, as well as of impaired HC-dependent memory and HC neurogenesis (Conboy et al., 2011). Taken together, this information suggests a role of MIF in mediating the antidepressant action of exercise, probably by enhancing 5-HT neurotransmission and neurogenesis. 


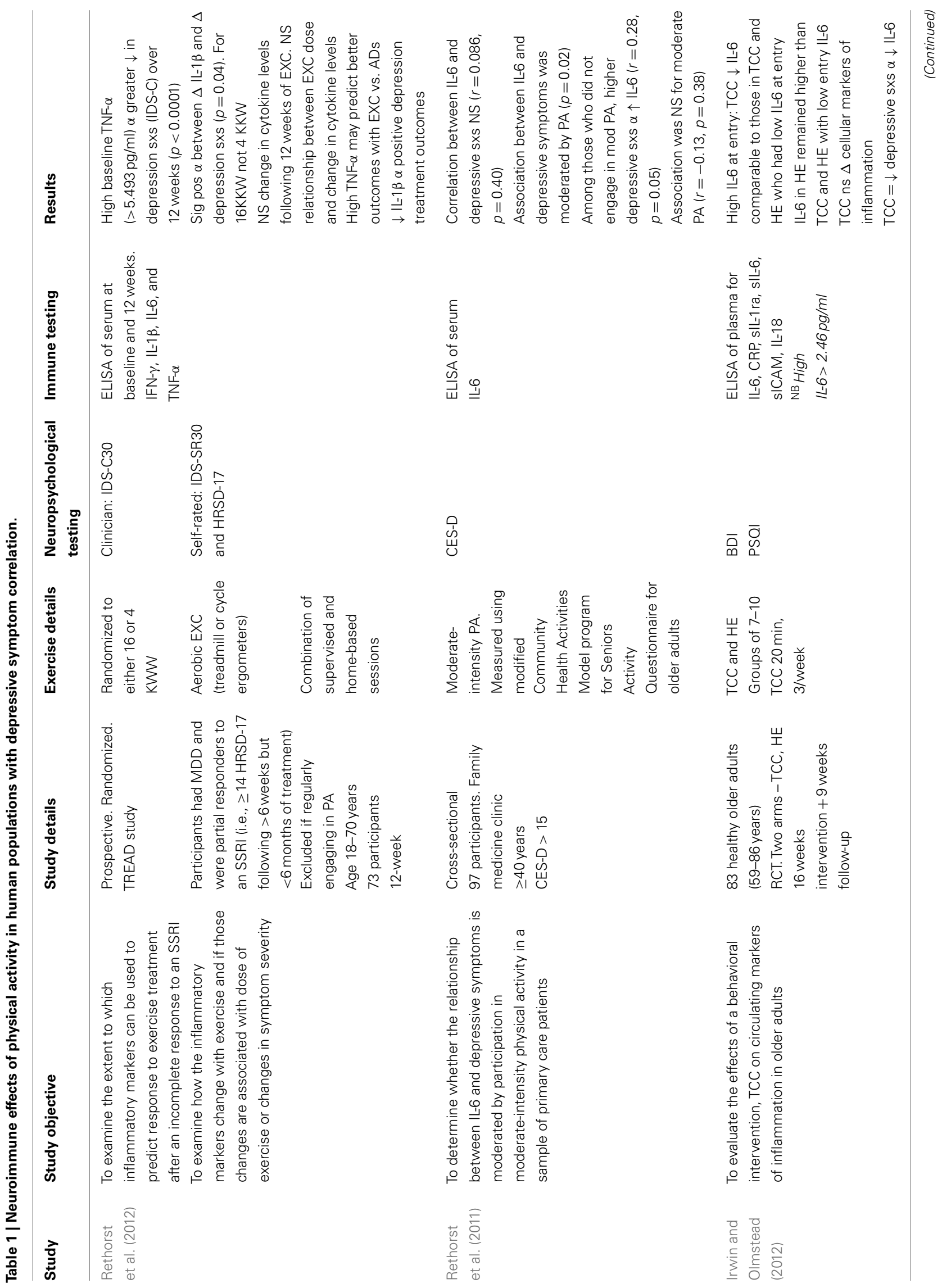




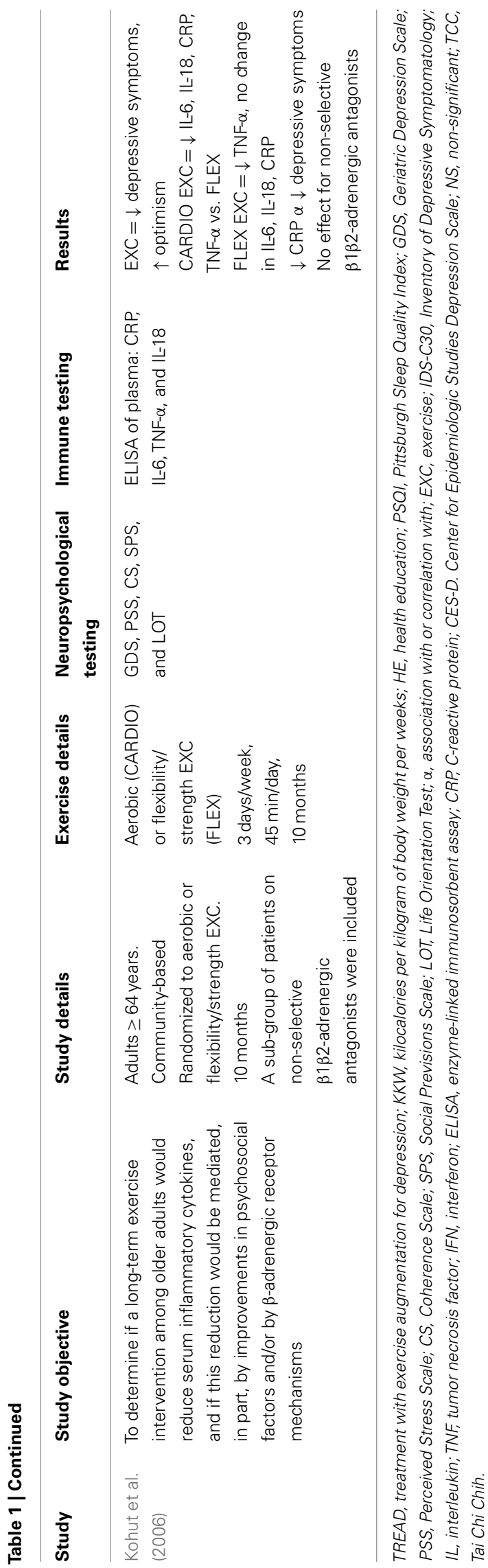

Other studies found investigating the effects of PA on neuroimmune-related factors suggest PA increases antiinflammatory or immunomodulatory factors, e.g., IL-10, IGF-1, and CX3CL1. Sigwalt et al. (2011) shows that in a rat model of depression induced by repeated dexamethasone administration, swimming exercise reduces depression-like behavior in correlation with increased HC IL-10, BDNF, and DNA oxidation. Duman et al. (2009) and Kohman et al. (2012) show voluntary wheel running associated with increased IGF-1, a factor recently shown to have anti-inflammatory effects.

Physical activity has been found to have beneficial effects on immunocompetent glial cells. A study by Latimer et al. (2011) has shown PA to revise age-related astrocyte hypertrophic/reactivity and myelin dysregulation - changes associated with neuroinflammation, cognitive decline, and reduced vascular function. Kohman et al. (2012) recently published a study showing PA attenuates aging associated increases in the proportion of new microglia within the HC (Iba-1 labeled). Furthermore, they show PA increases the pro-neurogenic phenotype of microglia (i.e., IGF-1-releasing microglia) which may contribute to increased HC neurogenesis. Given the robust anti-inflammatory effect of PA, the authors suggest PA may reduce PIC protein production leading to impaired microglial proliferation. A recent study by Barrientos et al. (2011) shows access to a running wheel reduced PIC expression from cultured microglia of aged rats. A recent study by Vukovic et al. (2012) suggests PA enhances the immunomodulatory factor CX3CL1 in the HC, with this associated with enhanced microglia-dependent neural precursor activity, as per the ex vivo neurosphere assay.

A study by Funk et al. (2011) demonstrates that PA can offer significant protection to the $\mathrm{HC}$ in a chemical-induced injury model [via trimethyltin (TMT)] that involves TNF receptor signaling. PA attenuated TMT-induced changes such as loss of DG neurons and microglial activation. Furthermore, PA was accompanied by a significant elevation in IL-6 and IL-1ra mRNA levels and repressed elevations in PICs and chemokines (CCL2 and CCL3). Interestingly, the investigators identified a functional role for IL-6 in neuroprotection given mice deficient in IL-6 (IL-6 knock-out) were not responsive to the neuroprotective effects of PA on the HC. The effects of PA and TMT on IL-6 downstream signal events differed at the level of STAT3 activation. The beneficial effects of acute spikes in IL-6 with PA is clearly a significant factor in the anti-inflammatory effect of PA. In a human study by Starkie et al. (2003), $3 \mathrm{~h}$ of cycling blunted the endotoxin-induced increase in circulating TNF- $\alpha$ levels, and this effect was mimicked by an IL-6 infusion. Further, this regulatory role of IL- 6 on TNF- $\alpha$ levels was demonstrated in anti-IL- 6 treated mice and IL- 6 knock-out mice (Mizuhara et al., 1994; Matthys et al., 1995). Whilst acute elevations in IL-6 are found throughout the body (Funk et al., 2011), a recent study shows a selective increase in IL-6 localized to the HC (Rasmussen et al., 2011).

Neuroimmune cells may also have a role in the beneficial effects of PA. A study by Ziv et al. (2006) found PA, a component of the EE protocol, was associated with enhanced $\mathrm{HC}$ neurogenesis alongside a neuroprotective microglia phenotype and in the presence of a T-cell population. The role of CNS-specific T cells in the neuroprotective effects of PA is suggested given severe combined 


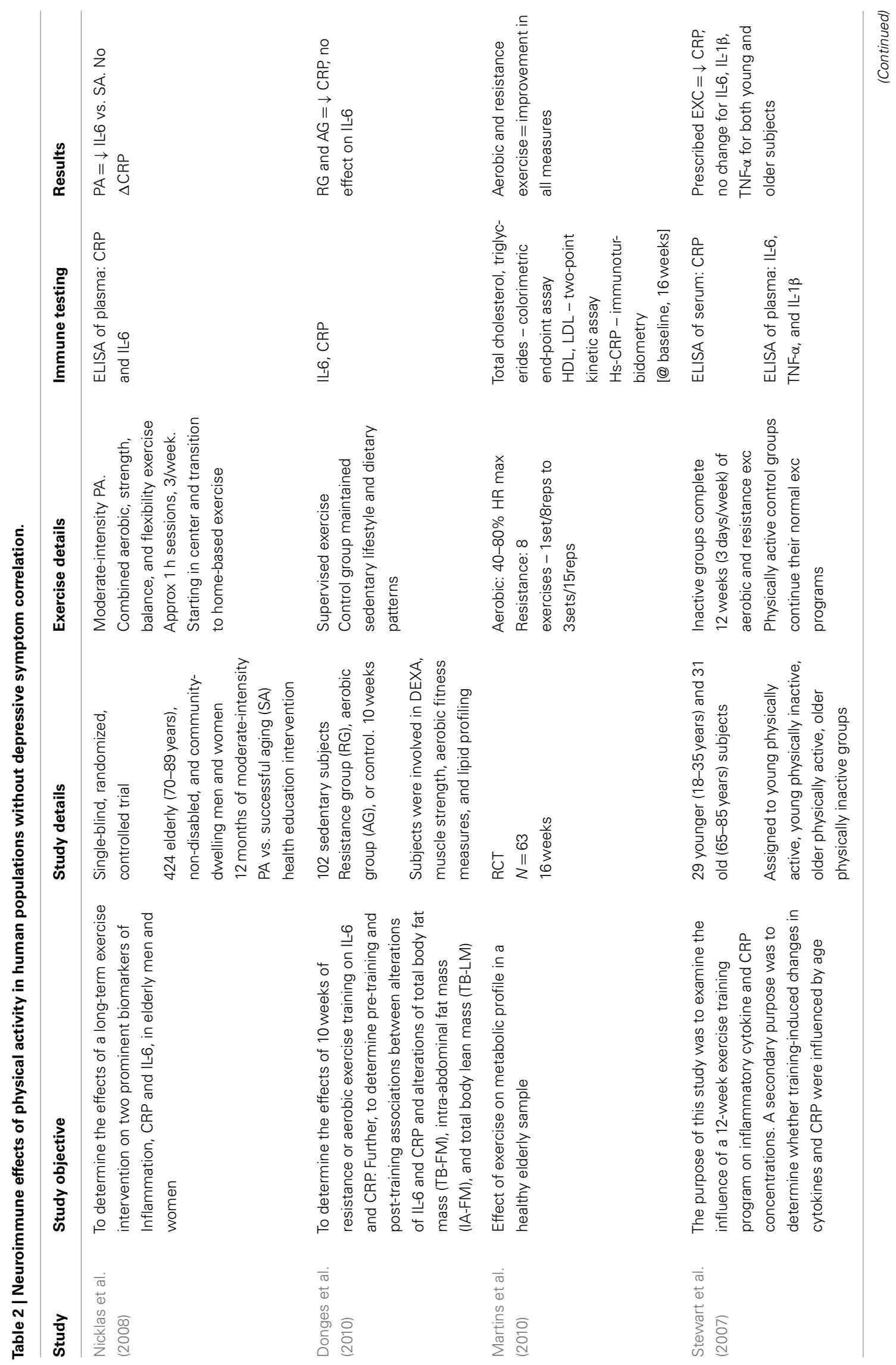



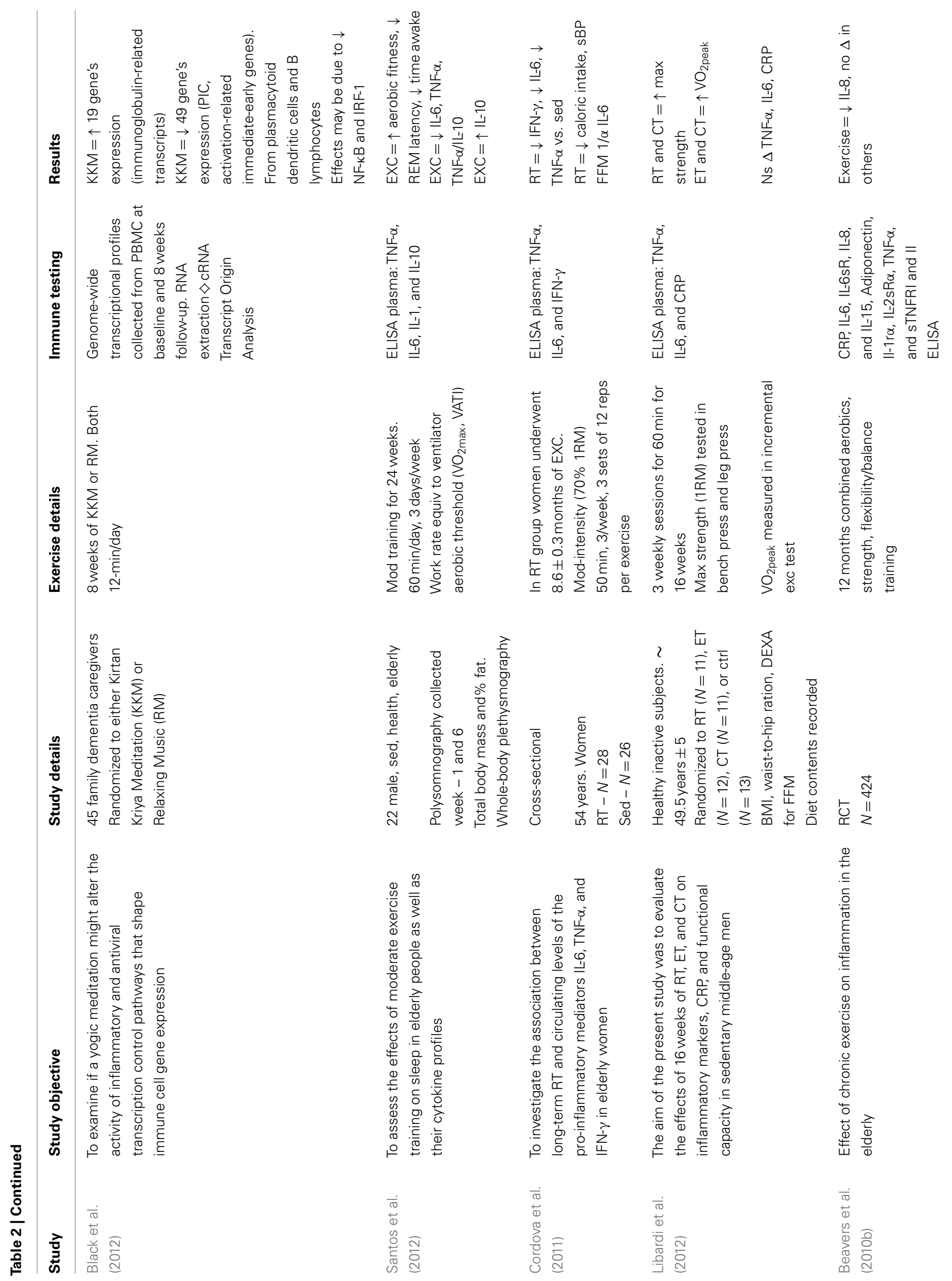

$\stackrel{\substack{4 \\ \text { I }}}{\mathbb{I}}$
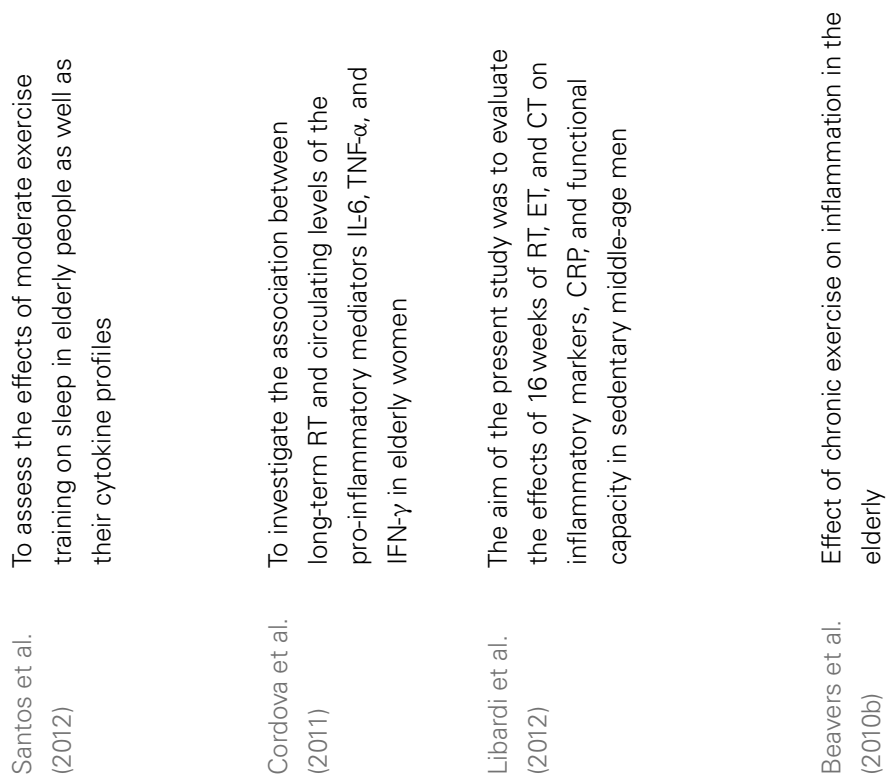


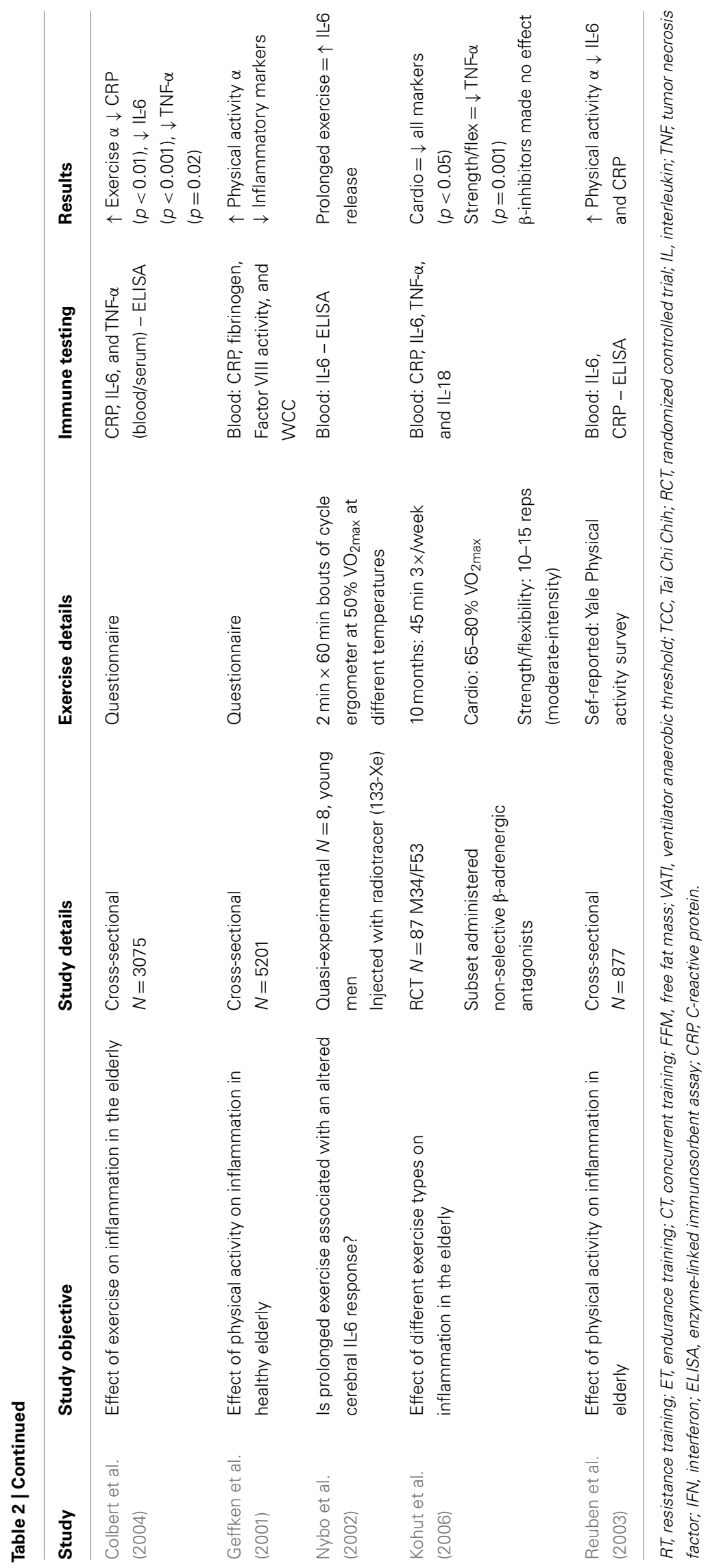




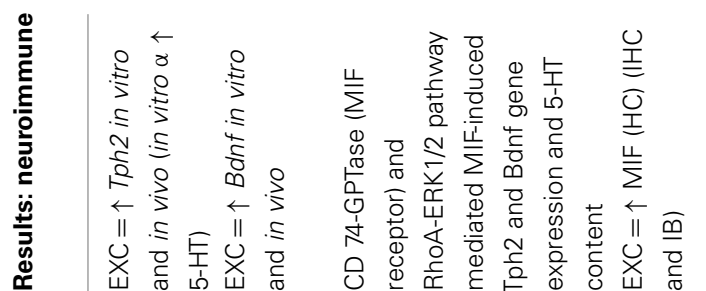

\|l

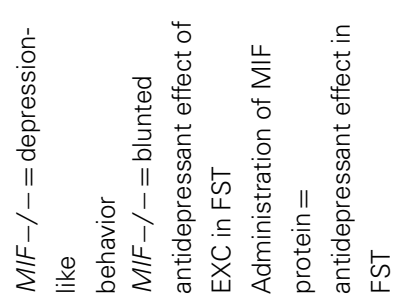

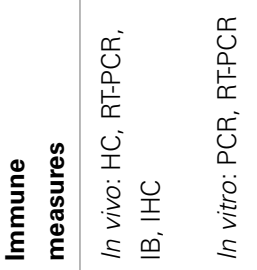

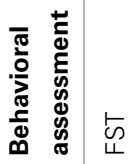

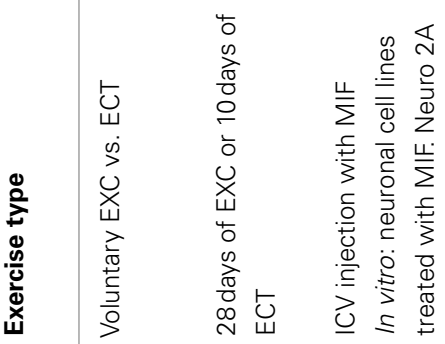

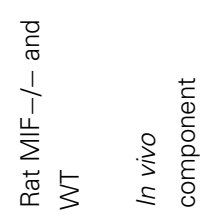

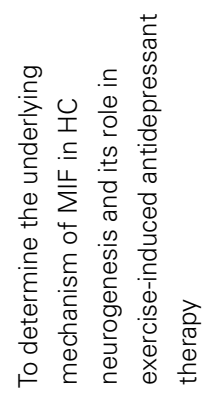

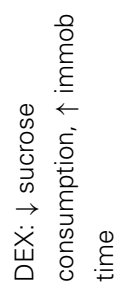

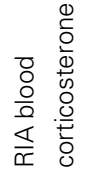

童

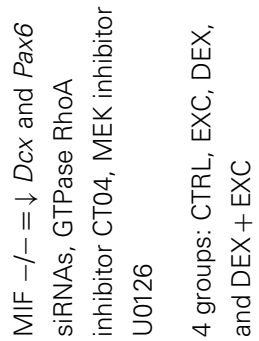

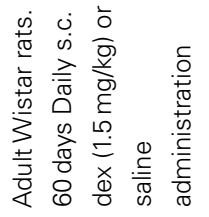

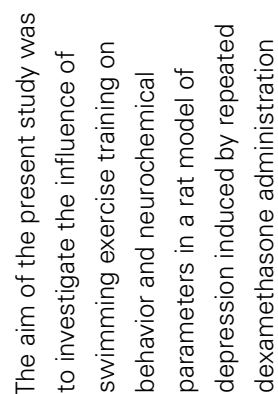

$\stackrel{5}{4}$

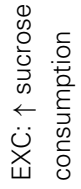

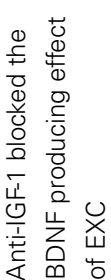
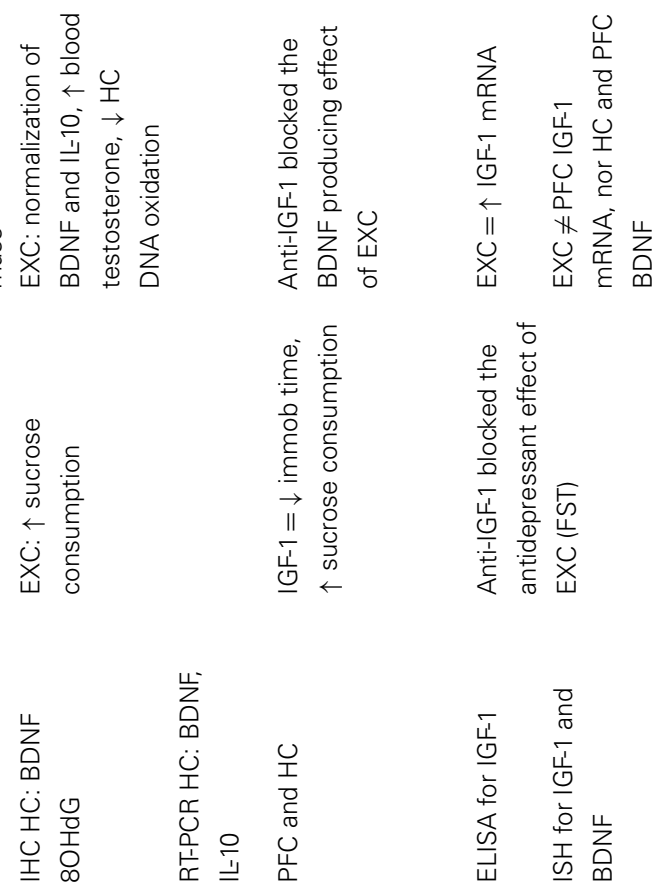

5

I

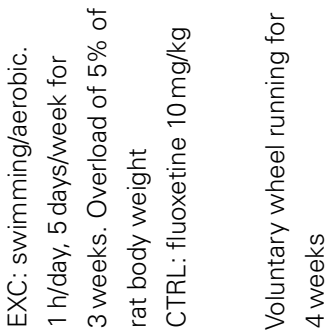

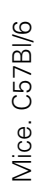

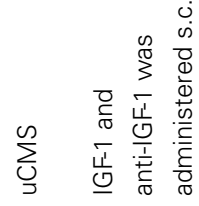
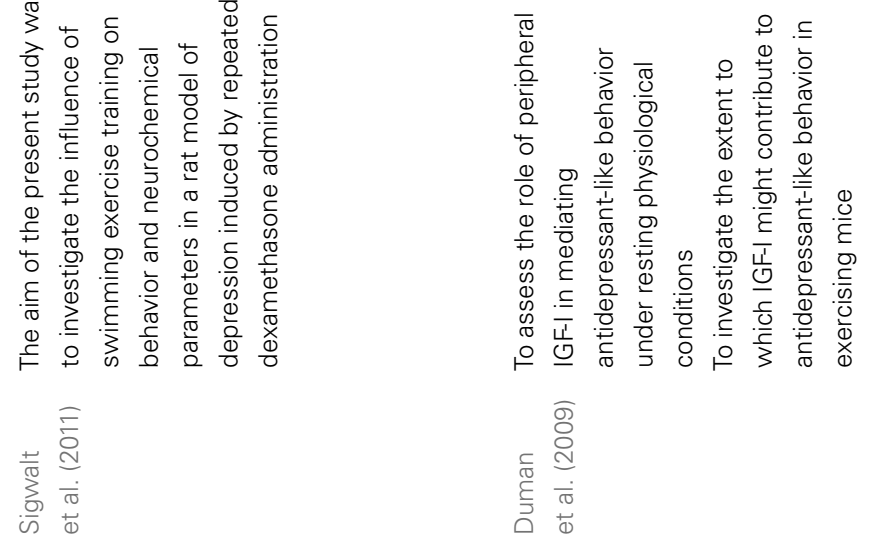

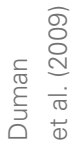

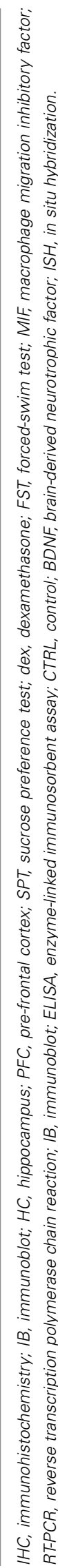




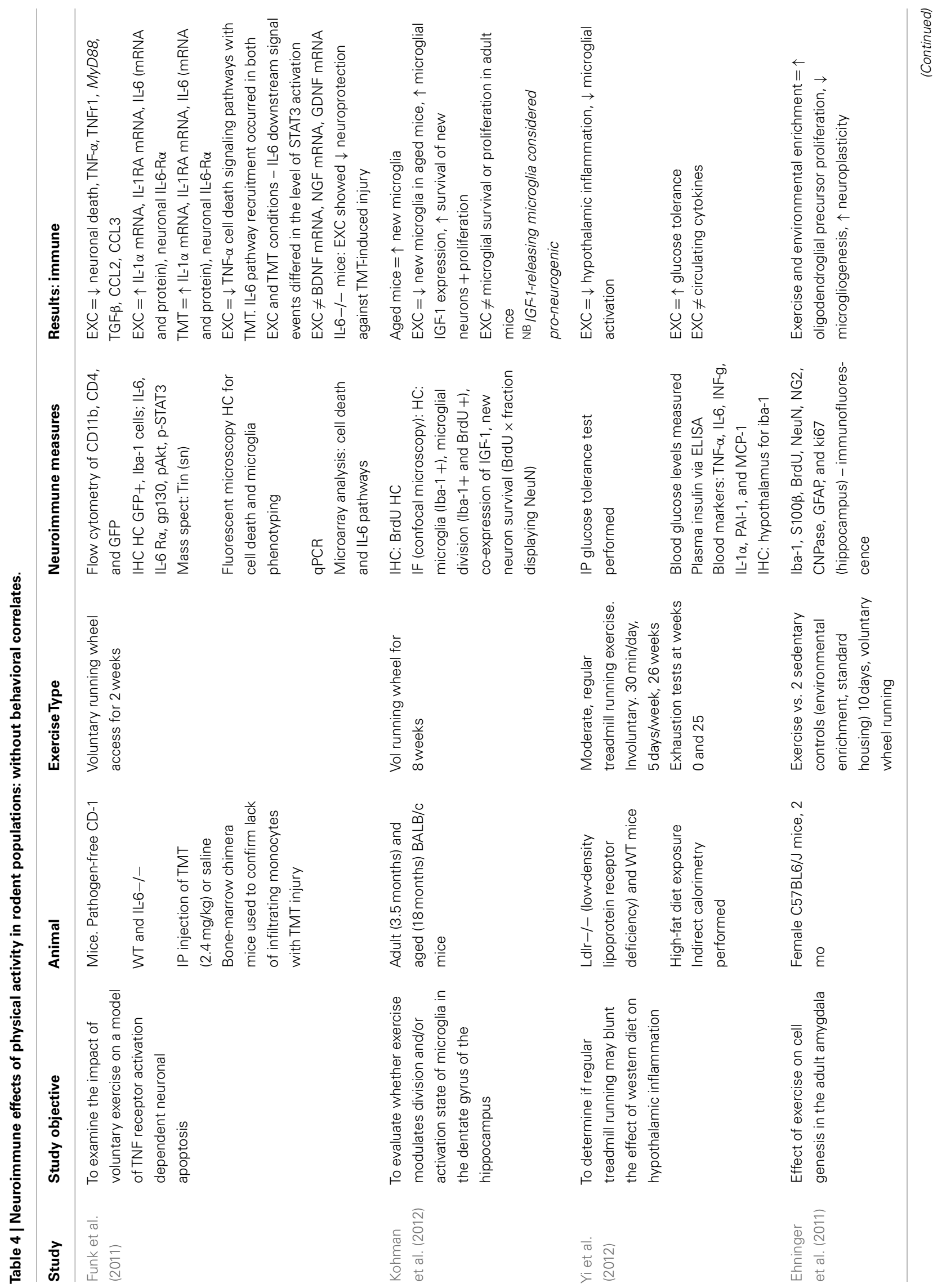




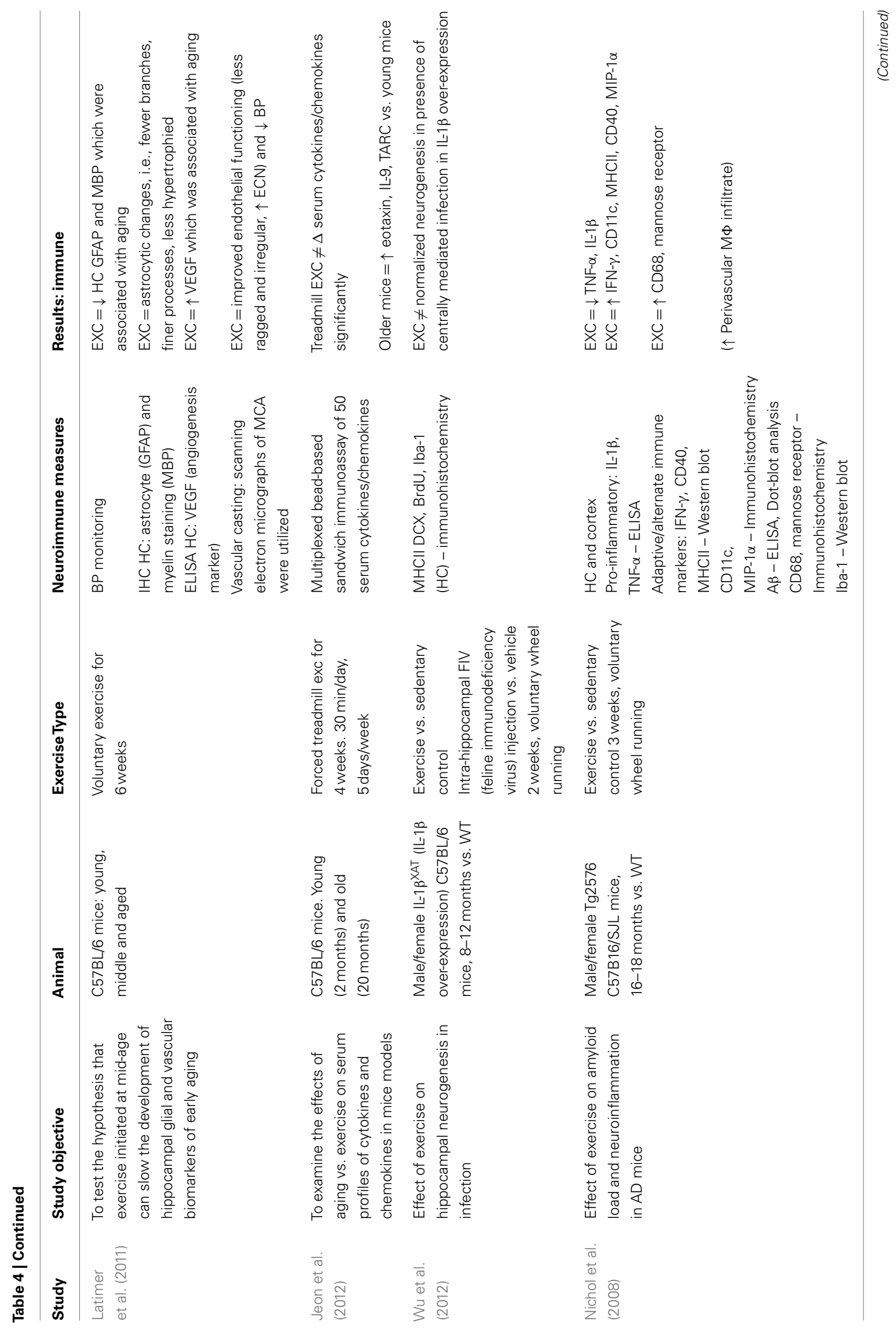




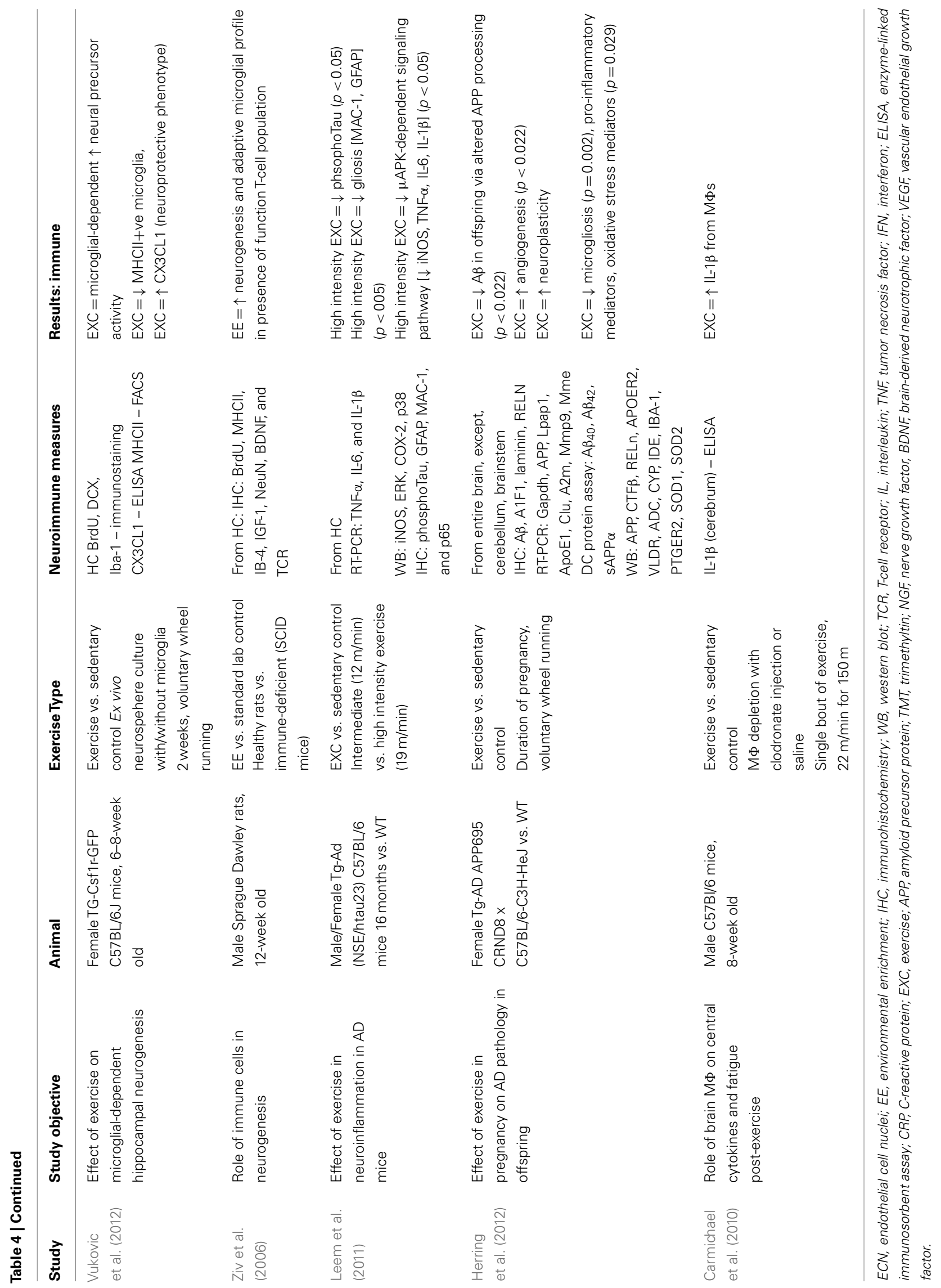


immunodeficiency (SCID) mice exposed to EE did not show an increase in neurogenesis.

\section{MODEL OF NEUROIMIMUNOLOGICAL EFFECTS OF PA IN DEPRESSION}

Emerging evidence suggests the neuroimmune system is critical in both the development of depression-related pathophysiology and in the treatment of depression. From the evidence available in this field, PA has a multitude of beneficial neuroimmune effects which may lead to the improvement of depression-related neurobiological processes, hence leading to reduced depression-like behaviors.

From a neuroimmune perspective, evidence suggests PA does enhance the beneficial and reduce the detrimental effects of the neuroimmune system. Figure 2 outlines these effects. PA appears to increase the following factors: IL-10, IL-6 (acutely), MIF, CNSspecific autoreactive CD4+ T cells, M2 microglia, quiescent astrocytes, CX3CL1, and IGF-1. On the other hand, PA appears to reduce detrimental neuroimmune factors such as: Th1/Th2 balance, PICs, CRP, M1 microglia, and reactive astrocytes. The effect of other factors is unknown, such as: T regs, CD200, chemokines, miRNA, M2-type blood-derived macrophages, and TNF- $\alpha$ (via R2). The beneficial effects of PA are likely to occur centrally and peripherally (e.g., in visceral fat reduction).

Based on the strong relationship between the neuroimmune system and other neurobiological systems (i.e., neuroplasticity, neuroendocrine function, and neurotransmission), we believe PA may exert beneficial behavioral effects via these neurobiological systems. PA's neuroimmune effects are likely involved in enhanced neuroplasticity, reduced oxidative stress, increases in 5HT, dopamine, and noradrenaline, and enhanced glucocorticoid sensitivity.

The neurobiological effects of PA - mediated largely via the neuroimmune system - are likely involved with reduced depression-like behaviors in rodents (i.e., sickness-like behavior, anhedonia, anxiety-, and cognition-like behaviors) and positive clinical effects (i.e., reduced depressive symptoms, enhanced cognitive function, relapse reduction, and early intervention).

\section{DISCUSSION}

Physical activity is increasingly investigated as a preventative, early intervention, and treatment option in depression. The interest in investigation of PA may have arisen for a number of reasons: the burden of depression is rising so novel therapeutic and preventative options are required (WHO, 2008; Berk and Jacka, 2012; Cuijpers et al., 2012; Southwick and Charney, 2012). Rates of physical inactivity are high and rising in modern society (Lee et al., 2012) with early evidence suggesting a link to the development of depression (Pasco et al., 2011a,b). Pharmacotherapy in depression is hampered by relatively high rates of resistance (Rush et al., 2006a,b) and considerable side-effects. Evidence is emerging to suggest co-morbid links between obesity, diabetes,

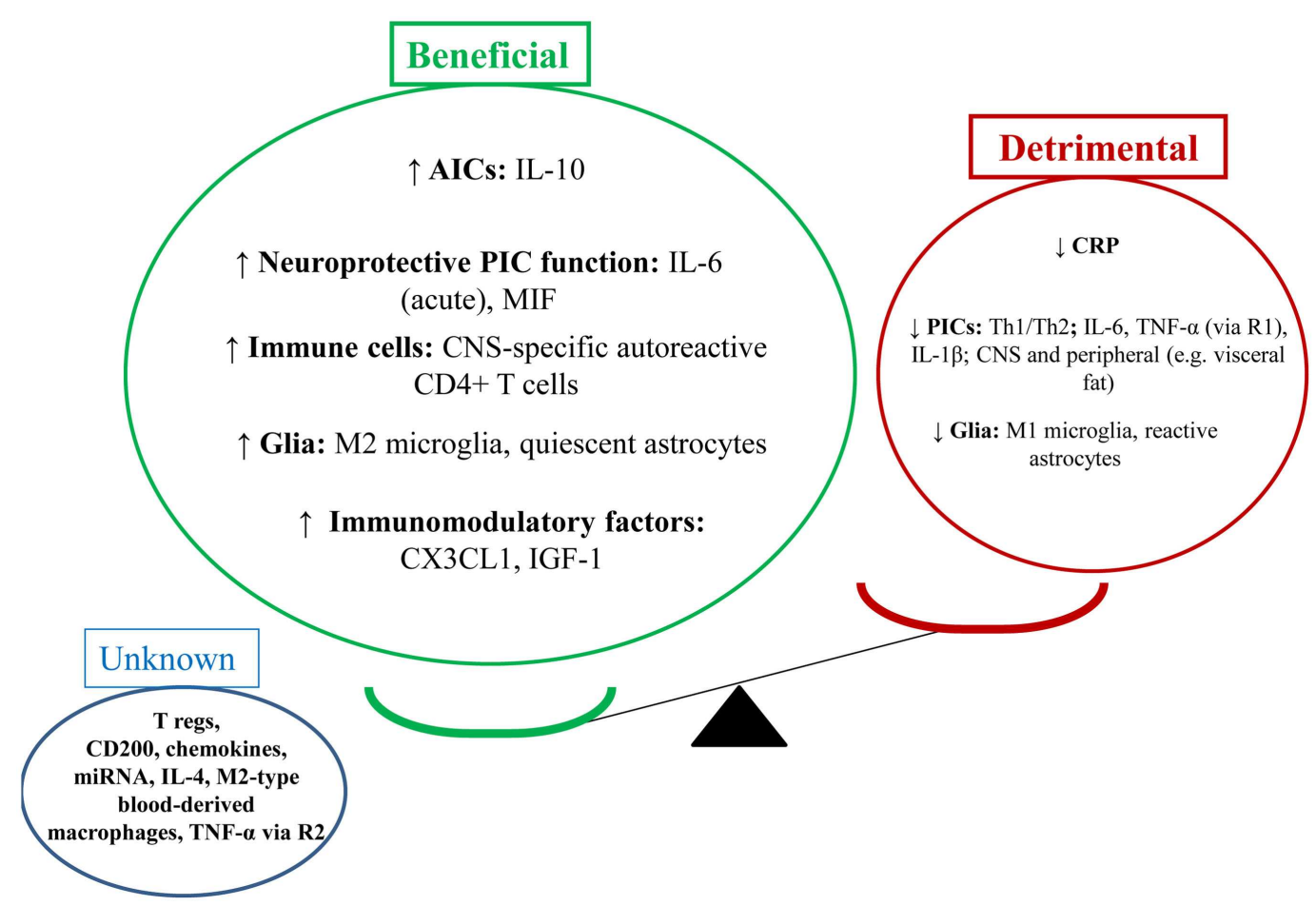

FIGURE 2 | Physical activity in depression: antidepressant via enhancing the beneficial effects of the neuroimmune system. This figure illustrates the effects of PA on the brain as per the balance between beneficial and detrimental effects of neuroimmune factors. PA appears to enhance the beneficial effects of the neuroimmune system and reduce the detrimental effects. From a behavioral perspective, this may lead to reduced depression-like behaviors. From a clinical perspective, this may lead to reduced depressive symptoms, depressive episode resolution, and reduced relapse rates (disease prevention). 
heart disease, and depression (Baune and Thome, 2011; Stuart and Baune, 2012), and PA is a therapeutic option with beneficial cardio-metabolic effects (Gleeson et al., 2011; Baune et al., 2012c; Hamer et al., 2012; Knochel et al., 2012; Stuart and Baune, 2012).

Based on the abovementioned factors, research has been reviewed to better understand the clinical efficacy of different types of PA, to understand the mechanism of action of PA and to investigate for suitable biomarkers to measure the treatment effect of PA in depression. Further, a model has been suggested in order to assist in understanding the neuroimmune effects of PA in depression.

An important consideration in the field of exercise immunology includes understanding the mechanisms of treatment response in depression vs. other psychiatric disorders. At present the authors feel there is no enough data to address this issue systematically, with research evidence. Whilst it would appear that the effects of PA on the immune system in various disorders - in both clinical and pre-clinical studies - is quite similar, i.e., PICs are reduced (particularly in anxiety disorders and depression; Gleeson et al., 2011; Eyre and Baune, 2012a), this considers only a narrow range of neuroimmune factors. The authors speculate that the therapeutic difference in PA may occur due to subtle variations in the neuroimmune and neurobiological effect, dependent upon the CNS environment with each pathophysiological state. Studies investigating the effects of a standardized exposure to PA, in various psychiatric disorders in parallel, may assist in unraveling this complex issue.

When considering the balance between the beneficial and detrimental effects of immune system and the effect of PA tipping this balance toward beneficial effects, it is important to consider: Is it possible to restore the balance of the immune system and still suffer from a low mood? This is an interesting question and open to debate. It would seem that the majority of evidence suggests that as inflammation increases, mood worsens, and as inflammation reduces, mood appears to return to normal. For example, this is shown in meta-analysis by Dowlati et al. (2010) and a review by Maes (2011) whereby depressive symptoms are associated with elevations in PIC levels. Another meta-analysis shows inflammation reduces with the use of SSRIs in the treatment of depression (Hannestad et al., 2011). However, there are other therapies such as SNRIs which appear to improve mood, yet have no effect on levels of inflammation (Hannestad et al., 2011). Therefore, more work is required to understand the effect of various therapies (pharmacological and non-pharmacological) on a wider variety of immune-related factors such as cytokines (anti- and pro-inflammatory), anti-inflammatory factors like IGF-1, CD200, CX3CL1, MIF, neuroprotective systemic immune cells, etc. Interestingly, Walker (2012) suggests the concentration of antidepressant drug molecules in the CNS also alters the immunomodulatory effects.

\section{FUTURE DIRECTIONS}

From current evidence, it is not possible to ascertain the type of PA which is most efficacious in the treatment of depression. Although, most evidence surrounds aerobic exercise. We suggest the need for head-to-head clinical trials comparing different types and intensities of PA to assist in making this issue clearer. Moreover, when considering the effects of distinct types of PA on neuroimmune factors, we also suggest the need for more head-to-head clinical trials (Baune and Eyre, 2012).

The most recent study examining the effects of PA on depressive symptoms was conducted by Rethorst et al. This study suggests that a high baseline TNF- $\alpha$ level was associated with a greater reduction in depressive symptomatology as opposed a high baseline TNF- $\alpha$ level being a negative factor for SSRI efficacy (Hannestad et al., 2011; Rethorst et al., 2012). The authors suggest TNF- $\alpha$ levels may be a moderator between SSRI and exercise treatment, and may have a role in personalized treatment algorithms. Whilst this is a promising suggestion, further research is needed to replicate these findings.

Our understanding of the neuroimmune effects of PA in depression will continue to develop as the understanding of the neuroimmune effects of PA develop. It is important to consider the use of multi-biomarker methods within this area in order to better understand potential biomarkers. For example, the use of neuroimaging, serum protein and genetic markers, and behavioral analysis. This type of methodology is increasingly employed in biological psychiatry (Baune et al., 2010, 2012a,b).

There are a number of neuroimmune-related factors which are yet to be considered in the effect of PA in depression. These factors include micro ribonucleic acid (miRNA), neuroimmune-related Positron Emission Tomography (PET) ligands, the neuroprotective effects of neuroimmune factors, and immune cells. Evidence is emerging to suggest a role for miRNAs, factors involved in regulating gene expression at the post-translational level, in modulating the effects of the immune system (Ponomarev et al., 2012). For example, various miRNAs such as miR-155 and miR-124 may have a role in polarizing microglia toward pro- or anti-inflammatory phenotypes, respectively (Ponomarev et al., 2012). The PET ligand, Translocator Protein (TPSO) ligand [(11)C]PBR28, a marker of microglial activation, was recently found to be elevated by LPSinduced systemic inflammation in non-human primates (Hannestad et al., 2012). This ligand has the potential to be utilized as a biomarker to investigate if activation of microglia may be a mechanism through which systemic inflammatory processes influence the disease course of depression. The biology of centrally migrating immune cells and CNS immune cells in depression is complex and far from understood. Regarding the debated issue of blood-derived macrophages can enter the brain parenchyma: research and development into novel methods for permanent differential labeling of circulating monocytes, as contrasted with resident microglia, is underway (Prinz et al., 2011). Studies are required to better understand the role of protective immunosurveillance in clinical and rodent models of depression.

\section{CONCLUSION}

The investigation of the neuroimmune effects of PA on depression and depression-like behavior is a rapidly developing and important field. This paper summarizes the most recent findings in the area and proposes a model whereby PA enhances the beneficial effects of the neuroimmune system and reduces the detrimental effects of the neuroimmune system. 


\section{REFERENCES}

Aarum, J., Sandberg, K., Haeberlein, S. L., and Persson, M. A. (2003). Migration and differentiation of neural precursor cells can be directed by microglia. Proc. Natl. Acad. Sci. U.S.A. 100, 15983-15988.

Anisman, H., Ravindran, A. V., Griffiths, J., and Merali, Z. (1999). Endocrine and cytokine correlates of major depression and dysthymia with typical or atypical features. Mol. Psychiatry 4, 182-188.

Araque, A., Parpura, V., Sanzgiri, R. P., and Haydon, P. G. (1999). Tripartite synapses: glia, the unacknowledged partner. Trends Neurosci. 22, 208-215.

Asmundson, G. J. G., Fetzner, M. G., DeBoer, L. B., Powers, M. B., Otto, M. W., and Smits, J. A. J. (2013). Let's get physical: a contemporary review of the anxiolytic effects of exercise for anxiety and its disorders. Depress. Anxiety. doi: 10.1002/da.22043. [Epub ahead of print].

Babyak, M., Blumenthal, J. A., Herman, S., Khatri, P., Doraiswamy, M., Moore, K., et al. (2000). Exercise treatment for major depression: maintenance of therapeutic benefit at 10 months. Psychosom. Med. 62, 633-638.

Bachstetter, A. D., Morganti, J. M., Jernberg, J., Schlunk, A., Mitchell, S. H., Brewster, K. W., et al. (2011). Fractalkine and CX 3 CR1 regulate hippocampal neurogenesis in adult and aged rats. Neurobiol. Aging 32, 2030-2044.

Bansal, A. S., Bradley, A. S., Bishop, K. N., Kiani-Alikhan, S., and Ford, B. (2012). Chronic fatigue syndrome, the immune system and viral infection. Brain Behav. Immun. 26, 24-31.

Barrientos, R. M., Frank, M. G., Crysdale, N. Y., Chapman, T. R., Ahrendsen, J. T., Day, H. E., et al. (2011). Little exercise, big effects: reversing aging and infection-induced memory deficits, and underlying processes. J. Neurosci. 31, 11578-11586.

Battista, D., Ferrari, C. C., Gage, F. H., and Pitossi, F. J. (2006). Neurogenic niche modulation by activated microglia: transforming growth factor beta increases neurogenesis in the adult dentate gyrus. Eur. J. Neurosci. 23, 83-93.

Baune, B. T., Dannlowski, U., Domschke, K., Janssen, D. G., Jordan, M. A., Ohrmann, P., et al. (2010). The interleukin 1 beta (IL1B) gene is associated with failure to achieve remission and impaired emotion processing in major depression. Biol. Psychiatry 67, 543-549.

Baune, B. T., and Eyre, H. (2012). Novel perspectives on the role of immune biomarkers in exercise and depression. Brain Behav. Immun. 26, 512.

Baune, B. T., Konrad, C., Grotegerd, D., Suslow, T., Birosova, E., Ohrmann, P., et al. (2012a). Interleukin-6 gene (IL-6): a possible role in brain morphology in the healthy adult brain. $J$. Neuroinflammation 9, 125.

Baune, B. T., Konrad, C., Grotegerd, D., Suslow, T., Ohrmann, P., Bauer, J., et al. (2012b). Tumor necrosis factor gene variation predicts hippocampus volume in healthy individuals. Biol. Psychiatry 72, 655-662.

Baune, B. T., Stuart, M., Gilmour, A., Wersching, H., Heindel, W., Arolt, V., et al. (2012c). The relationship between subtypes of depression and cardiovascular disease: a systematic review of biological models. Transl. Psychiatry 2, e92.

Baune, B. T., Ponath, G., Rothermundt, M., Riess, O., Funke, H., and Berger, K. (2008a). Association between genetic variants of IL-1beta, IL-6 and TNF-alpha cytokines and cognitive performance in the elderly general population of the MEMOstudy. Psychoneuroendocrinology 33, 68-76.

Baune, B. T., Wiede, F., Braun, A., Golledge, J., Arolt, V., and Koerner, H. (2008b). Cognitive dysfunction in mice deficient for TNF and its receptors. Am. J. Med. Genet. B Neuropsychiatr. Genet. 147B, 1056-1064.

Baune, B. T., and Thome, J. (2011). Translational research approach to biological and modifiable risk factors of psychosis and affective disorders. World J. Biol. Psychiatry 12(Suppl 1), 28-34.

Beavers, K. M., Brinkley, T. E., and Nicklas, B. J. (2010a). Effect of exercise training on chronic inflammation. Clin. Chim. Acta 411, 785-793.

Beavers, K. M., Hsu, F. C., Isom, S., Kritchevsky, S. B., Church, T., Goodpaster, B., et al. (2010b). Long-term physical activity and inflammatory biomarkers in older adults. Med. Sci. Sports Exerc. 42, 2189-2196.

Beers, D. R., Henkel, J. S., Zhao, W., Wang, J., and Appel, S. H. (2008). CD4+ $\mathrm{T}$ cells support glial neuroprotection, slow disease progression, and modify glial morphology in an animal model of inherited ALS. Proc. Natl. Acad. Sci. U.S.A. 105, 15558-15563.

Ben Menachem-Zidon, O., Avital, A., Ben-Menahem, Y., Goshen, I., Kreisel, T., Shmueli, E. M., et al. (2011). Astrocytes support hippocampal-dependent memory and long-term potentiation via interleukin-1 signaling. Brain Behav. Immun. 25, 1008-1016.

Berk, M., and Jacka, F. (2012). Preventive strategies in depression: gathering evidence for risk factors and potential interventions. Br. J. Psychiatry 201, 339-341.

Beumer, W., Gibney, S. M., Drexhage, R. C., Pont-Lezica, L., Doorduin, J., Klein, H. C., et al. (2012). The immune theory of psychiatric diseases: a key role for activated microglia and circulating monocytes. J. Leukoc. Biol. 92, 959-975.

Bilbo, S. D., Smith, S. H., and Schwarz, J. M. (2012). A lifespan approach to neuroinflammatory and cognitive disorders: a critical role for glia. J. Neuroimmune Pharmacol. 7, 24-41.

Black, D. S., Cole, S. W., Irwin, M. R. Breen, E., St Cyr, N. M., Nazarian, N., et al. (2012). Yogic meditation reverses NF-kappaB and IRF-related transcriptome dynamics in leukocytes of family dementia caregivers in a randomized controlled trial. Psychoneuroendocrinology. doi: 10.1016/j.psyneuen.2012.06.011. [Epub ahead of print].

Blume, J., Douglas, S. D., and Evans, D. L. (2011). Immune suppression and immune activation in depression. Brain Behav. Immun. 25, 221-229.

Blumenthal, J. A., Babyak, M. A., Moore, K. A., Craighead, W. E., Herman, S., Khatri, P., et al. (1999). Effects of exercise training on older patients with major depression. Arch. Intern. Med. 159, 2349-2356.

Blumenthal, J. A., Babyak, M. A., O'Connor, C., Keteyian, S., Landzberg, J., Howlett, J., et al. (2012a). Effects of exercise training on depressive symptoms in patients with chronic heart failure: the HF-action randomized trial. JAMA 308, 465-474.

Blumenthal, J. A., Sherwood, A., Babyak, M. A., Watkins, L. L., Smith, P. J., Hoffman, B. M., et al. (2012b). Exercise and pharmacological treatment of depressive symptoms in patients with coronary heart disease: results from the UPBEAT (understanding the prognostic benefits of exercise and antidepressant therapy) Study. J. Am. Coll. Cardiol. 60, 1053-1063.

Bluthe, R. M., Castanon, N., Pousset, F., Bristow, A., Ball, C., Lestage, J., et al. (1999). Central injection of IL-10 antagonizes the behavioural effects of lipopolysaccharide in rats. Psychoneuroendocrinology 24, 301-311.

Bowen, K. K., Dempsey, R. J., and Vemuganti, R. (2011). Adult interleukin-6 knockout mice show compromised neurogenesis. Neuroreport 22, 126-130.

Bracchi-Ricard, V., Brambilla, R., Levenson, J., Hu, W. H., Bramwell, A., Sweatt, J. D., et al. (2008). Astroglial nuclear factor-kappaB regulates learning and memory and synaptic plasticity in female mice. $J$. Neurochem. 104, 611-623.

Bridle, C., Spanjers, K., Patel, S., Atherton, N. M., and Lamb, S. E. (2012). Effect of exercise on depression severity in older people: systematic review and meta-analysis of randomised controlled trials. Br. J. Psychiatry 201, 180-185.

Brynskikh, A., Warren, T., Zhu, J., and Kipnis, J. (2008). Adaptive immunity affects learning behavior in mice. Brain Behav. Immun. 22, 861-869.

Butovsky, O., Koronyo-Hamaoui, M., Kunis, G., Ophir, E., Landa, G., Cohen, H., et al. (2006a). Glatiramer acetate fights against Alzheimer's disease by inducing dendritic-like microglia expressing insulin-like growth factor 1. Proc. Natl. Acad. Sci. U.S.A. 103, 11784-11789.

Butovsky, O., Ziv, Y., Schwartz, A., Landa, G., Talpalar, A. E., Pluchino, S., et al. (2006b). Microglia activated by IL-4 or IFN-gamma differentially induce neurogenesis and oligodendrogenesis from adult stem/progenitor cells. Mol. Cell. Neurosci. 31, 149-160.

Butovsky, O., Kunis, G., KoronyoHamaoui, M., and Schwartz, M. (2007). Selective ablation of bone marrow-derived dendritic cells increases amyloid plaques in a mouse Alzheimer's disease model. Eur. J. Neurosci. 26, 413-416.

Butovsky, O., Talpalar, A. E., BenYaakov, K., and Schwartz, M. (2005) Activation of microglia by aggregated beta-amyloid or lipopolysaccharide impairs MHC-II expression and renders them cytotoxic whereas IFN-gamma and IL-4 render them protective. Mol. Cell. Neurosci. 29, 381-393.

Butti, E., Bergami, A., Recchia, A., Brambilla, E., Del Carro, U., Amadio, S., et al. (2008). IL4 gene delivery to the CNS recruits regulatory $\mathrm{T}$ cells and induces clinical recovery in mouse models of multiple sclerosis. Gene Ther. 15, 504-515.

Capuron, L., and Miller, A. H. (2011). Immune system to brain signaling: neuropsychopharmacological implications. Pharmacol. Ther. 130, 226-238.

Cardon, M., Ron-Harel, N., Cohen, H., Lewitus, G. M., and Schwartz, M. (2010). Dysregulation of kisspeptin and neurogenesis at adolescence 
link inborn immune deficits to the late onset of abnormal sensorimotor gating in congenital psychological disorders. Mol. Psychiatry 15, 415-425.

Carlson, N. G., Wieggel, W. A., Chen, J., Bacchi, A., Rogers, S. W., and Gahring, L. C. (1999). Inflammatory cytokines IL-1 alpha, IL-1 beta, IL-6, and TNF-alpha impart neuroprotection to an excitotoxin through distinct pathways. J. Immunol. 163, 3963-3968.

Carmichael, M. D., Davis, J. M., Murphy, E. A., Carson, J. A., Van Rooijen, N., Mayer, E., et al. (2010). Role of brain macrophages on IL-1beta and fatigue following eccentric exercise-induced muscle damage. Brain Behav. Immun. 24, 564-568.

Chapoval, S., Dasgupta, P., Dorsey, N. J., and Keegan, A. D. (2010). Regulation of the $\mathrm{T}$ helper cell type 2 (Th2)/T regulatory cell (Treg) balance by IL-4 and STAT6. J. Leukoc. Biol. 87, 1011-1018.

Chiu, I. M., Chen, A., Zheng, Y., Kosaras, B., Tsiftsoglou, S. A., Vartanian, T. K., et al. (2008). T lymphocytes potentiate endogenous neuroprotective inflammation in a mouse model of ALS. Proc. Natl. Acad. Sci. U.S.A. 105, 17913-17918.

Cohen, H., Ziv, Y., Cardon, M., Kaplan, Z., Matar, M. A., Gidron, Y., et al. (2006). Maladaptation to mental stress mitigated by the adaptive immune system via depletion of naturally occurring regulatory CD4+CD25+ cells. J. Neurobiol. 66, 552-563.

Colbert, L. H., Visser, M., Simonsick, E. M., Tracy, R. P., Newman, A. B., Kritchevsky, S. B., et al. (2004). Physical activity, exercise, and inflammatory markers in older adults: findings from the Health, Aging and Body Composition Study. J. Am. Geriatr. Soc. 52, 1098-1104.

Conboy, L., Varea, E., Castro, J. E., Sakouhi-Ouertatani, H., Calandra, T., Lashuel, H. A., et al. (2011). Macrophage migration inhibitory factor is critically involved in basal and fluoxetine-stimulated adult hippocampal cell proliferation and in anxiety, depression, and memoryrelated behaviors. Mol. Psychiatry $16,533-547$.

Cordova, C., Lopes, E. S. F. Jr., Pires, A. S., Souza, V. C., Brito, C. J., Moraes, C. F., et al. (2011). Longterm resistance training is associated with reduced circulating levels of IL6, IFN-gamma and TNF-alpha in elderly women. Neuroimmunomodulation 18, 165-170.
Corona, A. W., Huang, Y., O'Connor, J. C., Dantzer, R., Kelley, K. W., Popovich, P. G., et al. (2010). Fractalkine receptor (CX3CR1) deficiency sensitizes mice to the behavioral changes induced by lipopolysaccharide. $J$. Neuroinflammation 7, 93.

Corona, A. W., Norden, D. M., Skendelas, J. P., Huang, Y., O'Connor, J. C., Lawson, M., et al. (2012). Indoleamine 2,3dioxygenase inhibition attenuates lipopolysaccharide induced persistent microglial activation and depressive-like complications in fractalkine receptor $(\mathrm{CX}(3) \mathrm{CR} 1)$ deficient mice. Brain Behav Immun. doi:10.1016/j.bbi.2012.08.008. [Epub ahead of print]

Costello, D. A., Lyons, A., Denieffe, S., Browne, T. C., Cox, F. F., and Lynch, M. A. (2011). Long term potentiation is impaired in membrane glycoprotein CD200-deficient mice: a role for Toll-like receptor activation. J. Biol. Chem. 286, 34722-34732.

Cox, F. F., Carney, D., Miller, A. M., and Lynch, M. A. (2012). CD200 fusion protein decreases microglial activation in the hippocampus of aged rats. Brain Behav. Immun. 26, 789-796.

Craft, L. (2005). Exercise and clinical depression: examining two psychological mechanisms. Psychol. Sport. Exerc. 6, 151-171.

Cuijpers, P., Beekman, A. T., and Reynolds, C. F. III. (2012). Preventing depression: a global priority. JAMA 307, 1033-1034.

Curtsinger, J. M., Schmidt, C. S., Mondino, A., Lins, D. C., Kedl, R. M., Jenkins, M. K., et al. (1999). Inflammatory cytokines provide a third signal for activation of naive CD4+ and CD8+ T cells. J. Immunol. 162, 3256-3262.

Dantzer, R., O'Connor, J. C., Freund, G. G., Johnson, R. W., and Kelley, K. W. (2008). From inflammation to sickness and depression: when the immune system subjugates the brain. Nat. Rev. Neurosci. 9, 46-56.

Dantzer, R., O'Connor, J. C., Lawson, M. A., and Kelley, K. W. (2011). Inflammation-associated depression: from serotonin to kynurenine. Psychoneuroendocrinology 36, 426-436.

Derecki, N. C., Cardani, A. N., Yang, C. H., Quinnies, K. M., Crihfield, A., Lynch, K. R., et al. (2010). Regulation of learning and memory by meningeal immunity: a key role for IL-4. J. Exp. Med. 207, 1067-1080.

Derecki, N. C., Quinnies, K. M., and Kipnis, J. (2011). Alternatively activated myeloid (M2) cells enhance cognitive function in immune compromised mice. Brain Behav. Immun. 25, 379-385.

Deslandes, A. C., Moraes, H., Alves, H., Pompeu, F. A., Silveira, H., Mouta, R., et al. (2010). Effect of aerobic training on EEG alpha asymmetry and depressive symptoms in the elderly: a 1-year follow-up study. Braz. J. Med. Biol. Res. 43, 585-592.

Dhabhar, F. S., Burke, H. M., Epel, E. S., Mellon, S. H., Rosser, R., Reus, V. I., et al. (2009). Low serum IL-10 concentrations and loss of regulatory association between IL- 6 and IL-10 in adults with major depression. $J$. Psychiatr. Res. 43, 962-969.

Diener, E. (1984). Subjective well-being. Psychol. Bull. 94, 542-575.

Donges, C. E., Duffield, R., and Drinkwater, E. J. (2010). Effects of resistance or aerobic exercise training on interleukin-6, C-reactive protein, and body composition. Med. Sci. Sports Exerc. 42, 304-313.

Dowlati, Y., Herrmann, N., Swardfager, W., Liu, H., Sham, L., Reim, E. K., et al. (2010). A meta-analysis of cytokines in major depression. Biol. Psychiatry 67, 446-457.

Duman, C. H., Schlesinger, L., Terwilliger, R., Russell, D. S., Newton, S. S., and Duman, R. S. (2009). Peripheral insulin-like growth factor-I produces antidepressant-like behavior and contributes to the effect of exercise. Behav. Brain Res. 198, 366-371.

Ehninger, D., Wang, L. P., Klempin, F., Romer, B., Kettenmann, H., and Kempermann, G. (2011). Enriched environment and physical activity reduce microglia and influence the fate of NG2 cells in the amygdala of adult mice. Cell Tissue Res. 345, 69-86.

Ekdahl, C. T. (2012). Microglial activation - tuning and pruning adult neurogenesis. Front. Pharmacol. 3:41. doi:10.3389/fphar.2012.00041

Eller, T., Vasar, V., Shlik, J., and Maron, E. (2008). Pro-inflammatory cytokines and treatment response to escitalopram in major depressive disorder. Prog. Neuropsychopharmacol. Biol. Psychiatry 32, 445-450.

Erickson, K. I., Miller, D. L., and Roecklein, K. A. (2012). The aging hippocampus: interactions between exercise, depression, and BDNF. Neuroscientist 18, 82-97.

Erickson, K. I., Voss, M. W., Prakash, R. S., Basak, C., Szabo, A., Chaddock, L., et al. (2011). Exercise training increases size of hippocampus and improves memory. Proc. Natl. Acad. Sci. U.S.A. 108, 3017-3022.
Eyre, H., and Baune, B. T. (2012a). Neuroimmunological effects of physical exercise in depression. Brain Behav. Immun. 26, 251-266.

Eyre, H., and Baune, B. T. (2012b). Neuroimmunomodulation in unipolar depression: a focus on chronobiology and chronotherapeutics. $J$. Neural Transm. 119, 1147-1166.

Eyre, H., and Baune, B. T. (2012c). Neuroplastic changes in depression: a role for the immune system. Psychoneuroendocrinology 37, 1397-1416.

Foster, P. P., Rosenblatt, K. P., and Kuljis, R. O. (2011). Exercise-induced cognitive plasticity, implications for mild cognitive impairment and Alzheimer's disease. Front. Neurol. 2:28. doi:10.3389/fneur.2011.00028

Fox, K. (2000). "The effects of exercise on self-perceptions and selfesteem," in Physical Activity and Psychological Well-Being, ed. F. K. Biddle Sjh (London: Routledge), 88-117.

Frank, M. G., Baratta, M. V., Sprunger, D. B., Watkins, L. R., and Maier, S. F. (2007). Microglia serve as a neuroimmune substrate for stressinduced potentiation of CNS proinflammatory cytokine responses. Brain Behav. Immun. 21, 47-59.

Fujio, K., Okamura, T., and Yamamoto, K. (2010). The Family of IL10-secreting CD4+ $\mathrm{T}$ cells. Adv. Immunol. 105, 99-130.

Funk, J. A., Gohlke, J., Kraft, A. D. McPherson, C. A., Collins, J. B., and Jean Harry, G. (2011). Voluntary exercise protects hippocampal neurons from trimethyltin injury: possible role of interleukin-6 to modulate tumor necrosis factor receptor-mediated neurotoxicity. Brain Behav. Immun. 25, 1063-1077.

Gallagher, P. J., Castro, V., Fava, M., Weilburg, J. B., Murphy, S. N., Gainer, V. S., et al. (2012). Antidepressant response in patients with major depression exposed to NSAIDs: a pharmacovigilance study. Am. J. Psychiatry 169, 1065-1072.

Garber, C. E., Blissmer, B., Deschenes, M. R., Franklin, B. A., Lamonte, M. J., Lee, I. M., et al. (2011). American College of Sports Medicine position stand. Quantity and quality of exercise for developing and maintaining cardiorespiratory, musculoskeletal, and neuromotor fitness in apparently healthy adults: guidance for prescribing exercise. Med. Sci. Sports Exerc. 43, 1334-1359.

Garg, S. K., Banerjee, R., and Kipnis, J. (2008). Neuroprotective immunity: $\mathrm{T}$ cell-derived glutamate endows astrocytes with a neuroprotective 
phenotype. J. Immunol. 180, 3866-3873.

Geffken, D. F., Cushman, M., Burke, G. L., Polak, J. F., Sakkinen, P. A., and Tracy, R. P. (2001). Association between physical activity and markers of inflammation in a healthy elderly population. Am. J. Epidemiol. 153, 242-250.

Giunti, D., Parodi, B., Usai, C., Vergani, L., Casazza, S., Bruzzone, S., et al. (2012). Mesenchymal stem cells shape microglia effector functions through the release of CX3CL1. Stem Cells 30, 2044-2053.

Gleeson, M., Bishop, N. C., Stensel, D. J., Lindley, M. R., Mastana, S. S., and Nimmo, M. A. (2011). The anti-inflammatory effects of exercise: mechanisms and implications for the prevention and treatment of disease. Nat. Rev. Immunol. 11, 607-615.

Godbout, K., Fenn, A., Huang, Y., and Gensel, J. (2012). Central interleukin-4 infusion after a peripheral lipopolysaccharide injection promotes a neuroprotective CNS environment with increased M2 microglia. Brain Behav. Immun. 26, S29-S30.

Hafner, S., Emeny, R. T., Lacruz, M. E., Baumert, J., Herder, C., Koenig, W., et al. (2011). Association between social isolation and inflammatory markers in depressed and nondepressed individuals: results from the MONICA/KORA study. Brain Behav. Immun. 25, 1701-1707.

Hamer, M., Sabia, S., Batty, G. D., Shipley, M. J., Tabak, A. G., SinghManoux, A., et al. (2012). Physical activity and inflammatory markers over 10 years: follow-up in men and women from the Whitehall II Cohort Study. Circulation 126, 928-933.

Hannestad, J., Dellagioia, N., and Bloch, M. (2011). The effect of antidepressant medication treatment on serum levels of inflammatory cytokines: a meta-analysis. Neuropsychopharmacology 36, 2452-2459.

Hannestad, J., Gallezot, J. D., Schafbauer, T., Lim, K., Kloczynski, T., Morris, E. D., et al. (2012). Endotoxin-induced systemic inflammation activates microglia: [(1)(1)C]PBR28 positron emission tomography in nonhuman primates. Neuroimage 63, 232-239.

Hashimoto, K. (2009). Emerging role of glutamate in the pathophysiology of major depressive disorder. Brain Res. Rev. 61, 105-123.

Hauben, E., Butovsky, O., Nevo, U., Yoles, E., Moalem, G., Agranov, E., et al. (2000). Passive or active immunization with myelin basic protein promotes recovery from spinal cord contusion. J. Neurosci. 20, 6421-6430.

Hein, A. M., and O'Banion, M. K. (2012). Neuroinflammation and cognitive dysfunction in chronic disease and aging. J. Neuroimmune Pharmacol. 7, 3-6.

Herring, A., Donath, A., Yarmolenko, M., Uslar, E., Conzen, C., Kanakis, D., et al. (2012). Exercise during pregnancy mitigates Alzheimerlike pathology in mouse offspring. FASEB J. 26, 117-128.

Himmerich, H., Milenovic, S., Fulda, S., Plumakers, B., Sheldrick, A. J., Michel, T. M., et al. (2010). Regulatory $\mathrm{T}$ cells increased while ILlbeta decreased during antidepressant therapy. J. Psychiatr. Res. 44, 1052-1057.

Hinwood, M., Morandini, J., Day, T. A., and Walker, F. R. (2012). Evidence that microglia mediate the neurobiological effects of chronic psychological stress on the Medial prefrontal cortex. Cereb. Cortex 22, 1442-1454.

Hoffman, B. M., Blumenthal, J. A., Babyak, M. A., Smith, P. J., Rogers, S. D., Doraiswamy, P. M., et al. (2008). Exercise fails to improve neurocognition in depressed middle-aged and older adults. Med. Sci. Sports Exerc. 40, 1344-1352.

Irwin, M. R., and Olmstead, R. (2012). Mitigating cellular inflammation in older adults: a randomized controlled trial of Tai Chi Chih. Am. J. Geriatr. Psychiatry 20, 764-772.

Jenkins, M. K., and Johnson, J. G. (1993). Molecules involved in T-cell costimulation. Curr. Opin. Immunol. 5, 361-367.

Jeon, H., Mun, G. I., and Boo, Y. C. (2012). Analysis of serum cytokine/chemokine profiles affected by aging and exercise in mice. Cytokine 60, 487-492.

Kaneko, M., Stellwagen, D., Malenka, R. C., and Stryker, M. P. (2008). Tumor necrosis factor-alpha mediates one component of competitive, experience-dependent plasticity in developing visual cortex. Neuron 58, 673-680.

Kessler, R. C., Berglund, P., Demler, O., Jin, R., Merikangas, K. R., and Walters, E. E. (2005). Lifetime prevalence and age-of-onset distributions of DSM-IV disorders in the National Comorbidity Survey Replication. Arch. Gen. Psychiatry 62, 593-602.

Kim, S. J., Lee, H., Joung, H. Y., Lee, G., Lee, H. J., Shin, M. K., et al. (2011). T-bet deficient mice exhibit resistance to stress-induced development of depression-like behaviors. $J$. Neuroimmunol. 240-241, 45-51.

Kim, S. J., Lee, H., Lee, G., Oh, S. J., Shin, M. K., Shim, I., et al. (2012). CD4+CD25+ regulatory $\mathrm{T}$ cell depletion modulates anxiety and depression-like behaviors in mice. PLoS ONE 7:e42054. doi:10.1371/journal.pone.0042054

Kipnis, J., Avidan, H., Caspi, R. R., and Schwartz, M. (2004a). Dual effect of $\mathrm{CD} 4+\mathrm{CD} 25+$ regulatory $\mathrm{T}$ cells in neurodegeneration: a dialogue with microglia. Proc. Natl. Acad. Sci. U.S.A. 101(Suppl 2), 14663-14669.

Kipnis, J., Cohen, H., Cardon, M., Ziv, Y., and Schwartz, M. (2004b). T cell deficiency leads to cognitive dysfunction: implications for therapeutic vaccination for schizophrenia and other psychiatric conditions. Proc. Natl. Acad. Sci. U.S.A. 101, 8180-8185.

Kipnis, J., Derecki, N. C., Yang, C., and Scrable, H. (2008). Immunity and cognition: what do agerelated dementia, HIV-dementia and 'chemo-brain' have in common? Trends Immunol. 29, 455-463.

Knochel, C., Oertel-Knochel, V., O’Dwyer, L., Prvulovic, D., Alves, G., Kollmann, B., et al. (2012). Cognitive and behavioural effects of physical exercise in psychiatric patients. Prog. Neurobiol. 96, 46-68.

Kohl, H. W. III, Craig, C. L., Lambert, E. V., Inoue, S., Alkandari, J. R., Leetongin, G., et al. (2012). The pandemic of physical inactivity: global action for public health. Lancet 380 , 294-305.

Kohm, A. P., McMahon, J. S., Podojil, J. R., Begolka, W. S., Degutes, M., Kasprowicz, D. J., et al. (2006). Cutting edge: anti-CD25 monoclonal antibody injection results in the functional inactivation, not depletion, of $\mathrm{CD} 4+\mathrm{CD} 25+\mathrm{T}$ regulatory cells. J. Immunol. 176, 3301-3305.

Kohman, R. A., Deyoung, E. K., Bhattacharya, T. K., Peterson, L. N., and Rhodes, J. S. (2012). Wheel running attenuates microglia proliferation and increases expression of a proneurogenic phenotype in the hippocampus of aged mice. Brain Behav. Immun. 26, 803-810.

Kohut, M. L., McCann, D. A., Russell, D. W., Konopka, D. N., Cunnick, J. E., Franke, W. D., et al. (2006). Aerobic exercise, but not flexibility/resistance exercise, reduces serum IL-18, CRP, and IL-6 independent of betablockers, BMI, and psychosocial factors in older adults. Brain Behav. Immun. 20, 201-209.
Kokaia, Z., Martino, G., Schwartz, M., and Lindvall, O. (2012). Crosstalk between neural stem cells and immune cells: the key to better brain repair? Nat. Neurosci. 15, 1078-1087.

Koronyo-Hamaoui, M., Ko, M. K., Koronyo, Y., Azoulay, D., Seksenyan, A., Kunis, G., et al. (2009). Attenuation of $\mathrm{AD}$-like neuropathology by harnessing peripheral immune cells: local elevation of IL-10 and MMP-9. J. Neurochem. 111, 1409-1424.

Latimer, C. S., Searcy, J. L., Bridges, M. T., Brewer, L. D., Popovic, J., Blalock, E. M., et al. (2011). Reversal of glial and neurovascular markers of unhealthy brain aging by exercise in middle-aged female mice. PLoS ONE 6:e26812. doi:10.1371/journal.pone. 0026812

Lautenschlager, N. T., Cox, K., and Cyarto, E.V. (2012). The influence of exercise on brain aging and dementia. Biochim. Biophys. Acta 1822, 474-481.

Lavretsky, H., Alstein, L. L., Olmstead, R. E., Ercoli, L. M., Riparetti-Brown, M., Cyr, N. S., et al. (2011). Complementary use of Tai Chi Chih augments escitalopram treatment of geriatric depression: a randomized controlled trial. Am. J. Geriatr. Psychiatry 19, 839-850.

Lee, H. B., and Lyketsos, C. G. (2003). Depression in Alzheimer's disease: heterogeneity and related issues. Biol. Psychiatry 54, 353-362.

Lee, I. M., Shiroma, E. J., Lobelo, F., Puska, P., Blair, S. N., and Katzmarzyk, P. T. (2012). Effect of physical inactivity on major noncommunicable diseases worldwide: an analysis of burden of disease and life expectancy. Lancet 380 , 219-229.

Leem, Y. H., Lee, Y. I., Son, H. J., and Lee, S. H. (2011). Chronic exercise ameliorates the neuroinflammation in mice carrying NSE/htau23. Biochem. Biophys. Res. Commun. 406, 359-365.

Leonard, B., and Maes, M. (2012). Mechanistic explanations how cell-mediated immune activation, inflammation and oxidative and nitrosative stress pathways and their sequels and concomitants play a role in the pathophysiology of unipolar depression. Neurosci. Biobehav. Rev. 36, 764-785.

Lepore, S. J. (1997). Expressive writing moderates the relation between intrusive thoughts and depressive symptoms. J. Pers. Soc. Psychol. 73, 1030-1037.

Lewitus, G. M., Cohen, H., and Schwartz, M. (2008). Reducing 
post-traumatic anxiety by immunization. Brain Behav. Immun. 22, 1108-1114.

Li, Y., Xiao, B., Qiu, W., Yang, L., Hu, B., Tian, X., et al. (2010). Altered expression of CD4+CD25+ regulatory T cells and its 5-HT(1a) receptor in patients with major depression disorder. J. Affect. Disord. 124, 68-75.

Libardi, C. A., De Souza, G. V., Cavaglieri, C. R., Madruga, V. A., and Chacon-Mikahil, M. P. (2012). Effect of resistance, endurance, and concurrent training on TNF-alpha, IL-6, and CRP. Med. Sci. Sports Exerc. 44, 50-56.

Littrell, J. L. (2012). Taking the perspective that a depressive state reflects inflammation: implications for the use of antidepressants. Front. Psychol. 3:297. doi:10.3389/fpsyg.2012.00297

Liu, D., Wang, Z., Liu, S., Wang, F., Zhao, S., and Hao, A. (2011). Anti-inflammatory effects of fluoxetine in lipopolysaccharide(LPS)stimulated microglial cells. $\mathrm{Neu}$ ropharmacology 61, 592-599.

Lyons, A., Downer, E. J., Crotty, S., Nolan, Y. M., Mills, K. H., and Lynch, M. A. (2007). CD200 ligand receptor interaction modulates microglial activation in vivo and in vitro: a role for IL-4. J. Neurosci. 27, 8309-8313.

Lyons, A., McQuillan, K., Deighan, B. F., O'Reilly, J. A., Downer, E. J., Murphy, A. C., et al. (2009). Decreased neuronal CD200 expression in IL4-deficient mice results in increased neuroinflammation in response to lipopolysaccharide. Brain Behav. Immun. 23, 1020-1027.

Maes, M. (2011). Depression is an inflammatory disease, but cellmediated immune activation is the key component of depression. Prog. Neuropsychopharmacol. Biol. Psychiatry 35, 664-675.

Mahendra, N., and Arkin, S. (2003). Effects of four years of exercise, language, and social interventions on Alzheimer discourse. J. Commun. Disord. 36, 395-422.

Marosi, K., Bori, Z., Hart, N., Sarga, L., Koltai, E., Radak, Z., et al. (2012). Long-term exercise treatment reduces oxidative stress in the hippocampus of aging rats. Neuroscience 226, 21-28.

Martino, G., Pluchino, S., Bonfanti, L., and Schwartz, M. (2011). Brain regeneration in physiology and pathology: the immune signature driving therapeutic plasticity of neural stem cells. Physiol. Rev. 91, 1281-1304.

Martins, R. A., Neves, A. P., CoelhoSilva, M. J., Verissimo, M. T., and
Teixeira, A. M. (2010). The effect of aerobic versus strength-based training on high-sensitivity C-reactive protein in older adults. Eur. J. Appl. Physiol. 110, 161-169.

Mathieu, P., Piantanida, A. P., and Pitossi, F. (2010). Chronic expression of transforming growth factorbeta enhances adult neurogenesis. Neuroimmunomodulation 17 , 200-201.

Matthys, P., Mitera, T., Heremans, H., Van Damme, J., and Billiau, A. (1995). Anti-gamma interferon and anti-interleukin-6 antibodies affect staphylococcal enterotoxin B-induced weight loss, hypoglycemia, and cytokine release in D-galactosamine-sensitized and unsensitized mice. Infect. Immun. 63, 1158-1164.

McAfoose, J., and Baune, B. T. (2009). Evidence for a cytokine model of cognitive function. Neurosci. Biobehav. Rev. 33, 355-366.

McNally, L., Bhagwagar, Z., and Hannestad, J. (2008). Inflammation, glutamate, and glia in depression: a literature review. CNS Spectr. 13, 501-510.

Mead, G. E., Morley, W., Campbell, P., Greig, C. A., McMurdo, M., and Lawlor, D. A. (2008). Exercise for depression. Cochrane Database Syst. Rev. 4, CD004366. doi: 10.1002/14651858.CD004366.pub3

Mesquita, A. R., Correia-Neves, M., Roque, S., Castro, A. G., Vieira, P., Pedrosa, J., et al. (2008). IL-10 modulates depressive-like behavior. J. Psychiatr. Res. 43, 89-97.

Miller, A. H. (2010). Depression and immunity: a role for $\mathrm{T}$ cells? Brain Behav. Immun. 24, 1-8.

Miller, A. H., Maletic, V., and Raison, C. L. (2009). Inflammation and its discontents: the role of cytokines in the pathophysiology of major depression. Biol. Psychiatry 65, 732-741.

Mitschelen, M., Yan, H., Farley, J. A., Warrington, J. P., Han, S., Herenu, C. B., et al. (2011). Long-term deficiency of circulating and hippocampal insulin-like growth factor I induces depressive behavior in adult mice: a potential model of geriatric depression. Neuroscience 185, 50-60.

Mizuhara, H., O’Neill, E., Seki, N., Ogawa, T., Kusunoki, C., Otsuka, K., et al. (1994). T cell activationassociated hepatic injury: mediation by tumor necrosis factors and protection by interleukin 6. J. Exp. Med. 179, 1529-1537.

Moon, H. Y., Kim, S. H., Yang, Y. R., Song, P., Yu, H. S., Park, H. G., et al. (2012). Macrophage migration inhibitory factor mediates the antidepressant actions of voluntary exercise. Proc. Natl. Acad. Sci. U.S.A. 109, 13094-13099.

Moon, M. L., McNeil, L. K., and Freund, G. G. (2011). Macrophages make me sick: how macrophage activation states influence sickness behavior. Psychoneuroendocrinology 36, 1431-1440.

Moron, J. A., Zakharova, I., Ferrer, J. V., Merrill, G. A., Hope, B., Lafer, E. M., et al. (2003). Mitogen-activated protein kinase regulates dopamine transporter surface expression and dopamine transport capacity. J. Neurosci. 23, 8480-8488.

Moylan, S., Maes, M., Wray, N. R., and Berk, M. (2012). The neuroprogressive nature of major depressive disorder: pathways to disease evolution and resistance, and therapeutic implications. Mol. Psychiatry. doi: 10.1038/mp.2012.33. [Epub ahead of print].

Muller, N., Myint, A. M., and Schwarz, M. J. (2011). Inflammatory biomarkers and depression. Neurotox. Res. 19, 308-318.

Musil, R., Schwarz, M. J., Riedel, M., Dehning, S., Cerovecki, A., Spellmann, I., et al. (2011). Elevated macrophage migration inhibitory factor and decreased transforming growth factor-beta levels in major depression - no influence of celecoxib treatment. J. Affect. Disord. 134, 217-225.

Myint, A. M., Leonard, B. E., Steinbusch, H. W., and Kim, Y. K. (2005). Th1, Th2, and Th3 cytokine alterations in major depression. J. Affect. Disord. 88, 167-173.

Nakajima, A., Yamada, K., Nagai, T., Uchiyama, T., Miyamoto, Y., Mamiya, T., et al. (2004). Role of tumor necrosis factor-alpha in methamphetamine-induced drug dependence and neurotoxicity. $J$. Neurosci. 24, 2212-2225.

Nguyen, K., D’Mello, C., Le, T., Urbanski, S., and Swain, M. G. (2012). Regulatory T cells suppress sickness behaviour development without altering liver injury in cholestatic mice. J. Hepatol. 56, 626-631.

Nichol, K. E., Poon, W. W., Parachikova, A. I., Cribbs, D. H., Glabe, C. G., and Cotman, C. W. (2008). Exercise alters the immune profile in $\mathrm{Tg} 2576$ Alzheimer mice toward a response coincident with improved cognitive performance and decreased amyloid. J. Neuroinflammation 5,13 .

Nicklas, B. J., Hsu, F. C., Brinkley, T. J., Church, T., Goodpaster, B. H.,
Kritchevsky, S. B., et al. (2008). Exercise training and plasma Creactive protein and interleukin-6 in elderly people. J. Am. Geriatr. Soc. 56, 2045-2052.

Nybo, L., Nielsen, B., Pedersen, B. K., Moller, K., and Secher, N. H. (2002). Interleukin-6 release from the human brain during prolonged exercise. J. Physiol. (Lond.) 542, 991-995.

Ojo, B., Rezaie, P., Gabbott, P. L., Davies, H., Colyer, F., Cowley, T. R., et al. (2012). Age-related changes in the hippocampus (loss of synaptophysin and glial-synaptic interaction) are modified by systemic treatment with an NCAM-derived peptide, FGL. Brain Behav. Immun. 26, 778-788.

Olah, M., Ping, G., De Haas, A. H., Brouwer, N., Meerlo, P., Van Der Zee, E. A., et al. (2009). Enhanced hippocampal neurogenesis in the absence of microglia $\mathrm{T}$ cell interaction and microglia activation in the murine running wheel model. Glia 57, 1046-1061.

Park, S. E., Dantzer, R., Kelley, K. W., and McCusker, R. H. (2011a). Central administration of insulinlike growth factor-I decreases depressive-like behavior and brain cytokine expression in mice. $J$. Neuroinflammation 8, 12.

Park, S. E., Lawson, M., Dantzer, R., Kelley, K. W., and McCusker, R. H. (2011b). Insulin-like growth factor-I peptides act centrally to decrease depression-like behavior of mice treated intraperitoneally with lipopolysaccharide. J. Neuroinflammation 8, 179.

Pasco, J. A., Jacka, F. N., Williams, L. J., Brennan, S. L., Leslie, E., and Berk M. (2011a). Don't worry, be active: positive affect and habitual physical activity. Aust. N. Z. J. Psychiatry 45, 1047-1052.

Pasco, J. A., Williams, L. J., Jacka, F. N., Henry, M. J., Coulson, C. E., Brennan, S. L., et al. (2011b). Habitual physical activity and the risk for depressive and anxiety disorders among older men and women. Int. Psychogeriatr. 23, 292-298.

Pereira, A. C., Huddleston, D. E., Brickman, A. M., Sosunov, A. A., Hen, R., McKhann, G. M., et al. (2007). An in vivo correlate of exercise-induced neurogenesis in the adult dentate gyrus. Proc. Natl. Acad. Sci. U.S.A. 104, 5638-5643.

Ponomarev, E. D., Veremeyko, T., and Weiner, H. L. (2012). MicroRNAs are universal regulators of differentiation, activation, and polarization of microglia and macrophages in 
normal and diseased CNS. Glia 61, 91-103.

Popoli, M., Yan, Z., McEwen, B. S., and Sanacora, G. (2012). The stressed synapse: the impact of stress and glucocorticoids on glutamate transmission. Nat. Rev. Neurosci. 13, 22-37.

Prinz, M., Priller, J., Sisodia, S. S., and Ransohoff, R. M. (2011). Heterogeneity of CNS myeloid cells and their roles in neurodegeneration. Nat. Neurosci. 14, 1227-1235.

Quan, N., and Banks, W. A. (2007). Brain-immune communication pathways. Brain Behav. Immun. 21, 727-735.

Raison, C. L., and Miller, A. H. (2011). Is depression an inflammatory disorder? Curr. Psychiatry Rep. 13, 467-475.

Raison, C. L., Rutherford, R. E., Woolwine, B. J., Shuo, C., Schettler, P., Drake, D. F., et al. (2012). A randomized controlled trial of the tumor necrosis factor antagonist infliximab for treatment-resistant depression: the role of baseline inflammatory biomarkers. Arch. Gen. Psychiatry. doi: 10.1001/2013.jamapsychiatry.4. [Epub ahead of print].

Ransohoff, R. M., Kivisakk, P., and Kidd, G. (2003). Three or more routes for leukocyte migration into the central nervous system. Nat. Rev. Immunol. 3, 569-581.

Rasmussen, P., Vedel, J. C., Olesen, J., Adser, H., Pedersen, M. V., Hart, E., et al. (2011). In humans IL-6 is released from the brain during and after exercise and paralleled by enhanced IL- 6 mRNA expression in the hippocampus of mice. Acta Physiol. (Oxf.) 201, 475-482.

Rethorst, C. D., Moynihan, J., Lyness, J. M., Heffner, K. L., and Chapman, B. P. (2011). Moderating effects of moderate-intensity physical activity in the relationship between depressive symptoms and interleukin- 6 in primary care patients. Psychosom. Med. 73, 265-269.

Rethorst, C. D., Toups, M. S., Greer, T. L., Nakonezny, P. A., Carmody, T. J., Grannemann, B. D., et al. (2012). Pro-inflammatory cytokines as predictors of antidepressant effects of exercise in major depressive disorder. Mol. Psychiatry. doi: 10.1038/mp.2012.125. [Epub ahead of print].

Rethorst, C. D., Wipfli, B. M., and Landers, D. M. (2009). The antidepressive effects of exercise: a metaanalysis of randomized trials. Sports Med. 39, 491-511.

Reuben, D. B., Judd-Hamilton, L., Harris, T. B., and Seeman, T. E. (2003). The associations between physical activity and inflammatory markers in high-functioning older persons: MacArthur Studies of Successful Aging. J. Am. Geriatr. Soc. 51, 1125-1130.

Rimer, J., Dwan, K., Lawlor, D. A., Greig, C. A., McMurdo, M., Morley, W., et al. (2012). Exercise for depression. Cochrane Database Syst. Rev. 7, CD004366.

Rogers, J. T., Morganti, J. M., Bachstetter, A. D., Hudson, C. E., Peters, M. M., Grimmig, B. A., et al. (2011). CX3CR1 deficiency leads to impairment of hippocampal cognitive function and synaptic plasticity. J. Neurosci. 31, 16241-16250.

Rolls, A., Schori, H., London, A., and Schwartz, M. (2008). Decrease in hippocampal neurogenesis during pregnancy: a link to immunity. Mol. Psychiatry 13, 468-469.

Ron-Harel, N., Cardon, M., and Schwartz, M. (2011). Brain homeostasis is maintained by "danger" signals stimulating a supportive immune response within the brain's borders. Brain Behav. Immun. 25, 1036-1043.

Rook, G. A., Lowry, C. A., and Raison, C. L. (2011). Lymphocytes in neuroprotection, cognition and emotion: is intolerance really the answer? Brain Behav. Immun. 25, 591-601.

Rush, A. J., Trivedi, M. H., Wisniewski, S. R., Nierenberg, A. A., Stewart, J. W., Warden, D., et al. (2006a). Acute and longer-term outcomes in depressed outpatients requiring one or several treatment steps: a STAR $* \mathrm{D}$ report. Am. J. Psychiatry 163, 1905-1917.

Rush, A. J., Trivedi, M. H., Wisniewski, S. R., Stewart, J. W., Nierenberg, A. A., Thase, M. E., et al. (2006b). Bupropion-SR, sertraline, or venlafaxine-XR after failure of SSRIs for depression. N. Engl. J. Med. 354, 1231-1242.

Salmon, P. (2001). Effects of physical exercise on anxiety, depression, and sensitivity to stress: a unifying theory. Clin. Psychol. Rev. 21, 33-61.

Santello, M., and Volterra, A. (2012). TNFalpha in synaptic function: switching gears. Trends Neurosci. 35 , 638-647.

Santos, R. V., Viana, V. A., Boscolo, R. A., Marques, V. G., Santana, M. G., Lira, F. S., et al. (2012). Moderate exercise training modulates cytokine profile and sleep in elderly people. Cytokine 60, 731-735.

Sarris, J., Moylan, S., Camfield, D. A., Pase, M. P., Mischoulon, D., Berk, M., et al. (2012). Complementary medicine, exercise, meditation, diet, and lifestyle modification for anxiety disorders: a review of current evidence. Evid. Based Complement. Alternat. Med. 2012, 809653.

Schwartz, M., Butovsky, O., Bruck, W., and Hanisch, U. K. (2006). Microglial phenotype: is the commitment reversible? Trends Neurosci. 29, 68-74.

Schwartz, M., and Shechter, R. (2010a). Protective autoimmunity functions by intracranial immunosurveillance to support the mind: the missing link between health and disease. Mol. Psychiatry 15, 342-354.

Schwartz, M., and Shechter, R. (2010b). Systemic inflammatory cells fight off neurodegenerative disease. Nat. Rev. Neurol. 6, 405-410.

Schwarz, J. M., and Bilbo, S. D. (2011). "The immune system and the developing brain," in Colloquium Series on the Developing Brain, Vol. 2, No. 3, ed. M. M. McCarthy (San Rafael: Morgan and Claypool Publishers), $1-128$.

Schwarz, J. M., and Bilbo, S. D. (2012). Sex, glia, and development: interactions in health and disease. Horm. Behav. 62, 243-253.

Shaked, I., Tchoresh, D., Gersner, R., Meiri, G., Mordechai, S., Xiao, X., et al. (2005). Protective autoimmunity: interferon-gamma enables microglia to remove glutamate without evoking inflammatory mediators. J. Neurochem. 92, 997-1009.

Shechter, R., London, A., Varol, C., Raposo, C., Cusimano, M., Yovel, G., et al. (2009). Infiltrating bloodderived macrophages are vital cells playing an anti-inflammatory role in recovery from spinal cord injury in mice. PLoS Med. 6:e1000113. doi:10.1371/journal.pmed.1000113

Sherry, C. L., Kim, S. S., Dilger, R. N., Bauer, L. L., Moon, M. L., Tapping, R. I., et al. (2010). Sickness behavior induced by endotoxin can be mitigated by the dietary soluble fiber, pectin, through up-regulation of IL4 and Th2 polarization. Brain Behav. Immun. 24, 631-640.

Shimizu, E., Kawahara, K., Kajizono, M., Sawada, M., and Nakayama, H. (2008). IL-4-induced selective clearance of oligomeric beta-amyloid peptide(1-42) by rat primary type 2 microglia. J. Immunol. 181, 6503-6513.

Sigwalt, A. R., Budde, H., Helmich, I., Glaser, V., Ghisoni, K., Lanza, S., et al. (2011). Molecular aspects involved in swimming exercise training reducing anhedonia in a rat model of depression. Neuroscience 192, 661-674.
Smith, P. J., Blumenthal, J. A., Hoffman, B. M., Cooper, H., Strauman, T. A., Welsh-Bohmer, K., et al. (2010). Aerobic exercise and neurocognitive performance: a meta-analytic review of randomized controlled trials. Psychosom. Med. 72, 239-252.

Southwick, S. M., and Charney, D. S. (2012). The science of resilience: implications for the prevention and treatment of depression. Science 338, 79-82.

Starkie, R., Ostrowski, S. R., Jauffred, S., Febbraio, M., and Pedersen, B. K. (2003). Exercise and IL-6 infusion inhibit endotoxin-induced TNF-alpha production in humans. FASEB J. 17, 884-886.

Stellwagen, D., and Malenka, R. C. (2006). Synaptic scaling mediated by glial TNF-alpha. Nature 440, 1054-1059.

Stewart, L. K., Flynn, M. G., Campbell, W. W., Craig, B. A., Robinson, J. P., Timmerman, K. L., et al. (2007). The influence of exercise training on inflammatory cytokines and Creactive protein. Med. Sci. Sports Exerc. 39, 1714-1719.

Stuart, M. J., and Baune, B. T. (2012). Depression and type 2 diabetes: inflammatory mechanisms of a psychoneuroendocrine comorbidity. Neurosci. Biobehav. Rev. $36,658-676$.

Sutcigil, L., Oktenli, C., Musabak, U., Bozkurt, A., Cansever, A., Uzun, O., et al. (2007). Pro- and antiinflammatory cytokine balance in major depression: effect of sertraline therapy. Clin. Dev. Immunol. 2007, 76396.

Trejo, J. L., Piriz, J., Llorens-Martin, M. V., Fernandez, A. M., Bolos, M., Leroith, D., et al. (2007). Central actions of liver-derived insulin-like growth factor I underlying its procognitive effects. Mol. Psychiatry 12, 1118-1128

Trivedi, M. H., Greer, T. L., Church, T. S., Carmody, T. J., Grannemann, B. D., Galper, D. I., et al. (2011). Exercise as an augmentation treatment for nonremitted major depressive disorder: a randomized, parallel dose comparison. J. Clin. Psychiatry 72, 677-684.

Trivedi, M. H., Rush, A. J., Wisniewski, S. R., Nierenberg, A. A., Warden, D., Ritz, L., et al. (2006). Evaluation of outcomes with citalopram for depression using measurementbased care in STAR $* \mathrm{D}$ : implications for clinical practice. Am. J. Psychiatry $163,28-40$.

Tse, H. M., Milton, M. J., Schreiner, S., Profozich, J. L., Trucco, M., and Piganelli, J. D. (2007). Disruption of 
innate-mediated proinflammatory cytokine and reactive oxygen species third signal leads to antigen-specific hyporesponsiveness. J. Immunol. 178, 908-917.

Vukovic, J., Colditz, M. J., Blackmore, D. G., Ruitenberg, M. J., and Bartlett, P. F. (2012). Microglia modulate hippocampal neural precursor activity in response to exercise and aging. $J$. Neurosci. 32, 6435-6443.

Walker, F. R. (2012). A review of the mechanism of action for selective serotonin reuptake inhibitors: do these drugs possess antiinflammatory properties and is this relevant in the treatment of depression? Neuropharmacology 67C, 304-317.

Walker, P. A., Letourneau, P. A., Bedi, S., Shah, S. K., Jimenez, F., and Cox, C. S. Jr (2011). Progenitor cells as remote "bioreactors": neuroprotection via modulation of the systemic inflammatory response. World J. Stem Cells 3, 9-18.

Walsh, J. T., and Kipnis, J. (2011). Regulatory T cells in CNS injury: the simple, the complex and the confused. Trends. Mol. Med. 17, 541-547.
Walton, N. M., Sutter, B. M., Laywell, E. D., Levkoff, L. H., Kearns, S. M., Marshall, G. P. II, et al. (2006). Microglia instruct subventricular zone neurogenesis. Glia 54, 815-825.

Warner-Schmidt, J. L., Vanover, K. E., Chen, E. Y., Marshall, J. J. and Greengard, P. (2011). Antidepressant effects of selective serotonin reuptake inhibitors (SSRIs) are attenuated by antiinflammatory drugs in mice and humans. Proc. Natl. Acad. Sci. U.S.A. 108, 9262-9267.

WHO. (2008). Global Burden of Disease: 2004 Update. Geneva: WHO.

Wong, M. L., Dong, C., Maestre-Mesa, J., and Licinio, J. (2008). Polymorphisms in inflammation-related genes are associated with susceptibility to major depression and antidepressant response. Mol. Psychiatry 13, 800-812.

Wu, M. D., Hein, A. M., Moravan, M. J., Shaftel, S. S., Olschowka, J. A., and O'Banion, M. K. (2012). Adult murine hippocampal neurogenesis is inhibited by sustained ILlbeta and not rescued by voluntary running. Brain Behav. Immun. 26, 292-300.

Yi, C. X., Al-Massadi, O., Donelan, E. Lehti, M., Weber, J., Ress, C., et al. (2012). Exercise protects against high-fat diet-induced hypothalamic inflammation. Physiol. Behav. 106, 485-490.

Yirmiya, R., and Goshen, I. (2011). Immune modulation of learning, memory, neural plasticity and neurogenesis. Brain Behav. Immun 25, 181-213.

Zhou, X., Bailey-Bucktrout, S., Jeker, L. T., and Bluestone, J. A. (2009). Plasticity of CD4+ FoxP3+ T cells. Curr. Opin. Immunol. 21, 281-285.

Zhu, C. B., Blakely, R. D., and Hewlett, W. A. (2006). The proinflammatory cytokines interleukin-1beta and tumor necrosis factor-alpha activate serotonin transporters. Neuropsychopharmacology 31, 2121-2131.

Ziv, Y., Ron, N., Butovsky, O., Landa G., Sudai, E., Greenberg, N., et al. (2006). Immune cells contribute to the maintenance of neurogenesis and spatial learning abilities in adulthood. Nat. Neurosci. 9, 268-275.
Conflict of Interest Statement: The authors declare that the research was conducted in the absence of any commercial or financial relationships that could be construed as a potential conflict of interest.

Received: 30 November 2012; paper pending published: 25 December 2012; accepted: 07 January 2013; published online: 04 February 2013.

Citation: Eyre HA, Papps $E$ and Baune BT (2013) Treating depression and depression-like behavior with physical activity: an immune perspective. Front. Psychiatry 4:3. doi: 10.3389/fpsyt.2013.00003

This article was submitted to Frontiers in Affective Disorders and Psychosomatic Research, a specialty of Frontiers in Psychiatry.

Copyright (C) 2013 Eyre, Papps and Baune. This is an open-access article distributed under the terms of the Creative Commons Attribution License, which permits use, distribution and reproduction in other forums, provided the original authors and source are credited and subject to any copyright notices concerning any third-party graphics etc. 